\title{
Chemical abundances in spiral and irregular galaxies
}

\section{$\mathrm{O}$ and $\mathrm{N}$ abundances derived from global emission-line spectra}

\author{
L. S. Pilyugin ${ }^{1,2}$, T. Contini ${ }^{2}$, and J. M. Vílchez ${ }^{3}$ \\ 1 Main Astronomical Observatory of National Academy of Sciences of Ukraine, 27 Zabolotnogo str., 03680 Kiev, Ukraine \\ e-mail: pilyugin@mao.kiev.ua \\ 2 Laboratoire d'Astrophysique de l'Observatoire Midi-Pyrénées, UMR 5572, 14 avenue E. Belin, 31400 Toulouse, France \\ e-mail: contini@ast.obs-mip.fr \\ ${ }^{3}$ Instituto de Astrofísica de Andalucía, CSIC, Apdo 3004, 18080 Granada, Spain \\ e-mail: jvm@iaa.es
}

Received 25 November 2003 / Accepted 4 April 2004

\begin{abstract}
The validity of oxygen and nitrogen abundances derived from the global emission-line spectra of galaxies via the $P$-method has been investigated using a collection of published spectra of individual $\mathrm{H}_{\text {II }}$ regions in irregular and spiral galaxies. The conclusion of Kobulnicky et al. (1999, ApJ, 514, 544) that global emission-line spectra can reliably indicate the chemical properties of galaxies has been confirmed. It has been shown that the comparison of the global spectrum of a galaxy with a collection of spectra of individual $\mathrm{H}_{\text {II }}$ regions can be used to distinguish high and low metallicity objects and to estimate accurate chemical abundances in a galaxy. The oxygen and nitrogen abundances in samples of UV-selected and normal nearby galaxies have been determined. It has been found that the UV-selected galaxies occupy the same area in the N/O-O/H diagram as individual $\mathrm{H}_{\text {II }}$ regions in nearby galaxies. Finally, we show that intermediate-redshift galaxies systematically deviate from the metallicity-luminosity trend of local galaxies.
\end{abstract}

Key words. galaxies: abundances - galaxies: ISM - galaxies: evolution

\section{Introduction}

Investigating the variations of chemical properties among galaxies is very important to understand their structure and evolution. Abundance determinations in spiral galaxies are based on the spectrophotometry of individual $\mathrm{H}_{\text {II }}$ regions. Good spectrophotometry of $\mathrm{H}$ II regions is now available for a large number of galaxies, and the reliability of chemical abundance determinations is defined mainly by the method used for abundance determinations in $\mathrm{H}_{\text {II }}$ regions. Accurate abundances in $\mathrm{H}$ II regions can be derived through the classical $T_{\mathrm{e}}$-method if measurements of temperature-sensitive line ratios are available. Unfortunately, in oxygen-rich $\mathrm{H}_{\text {II }}$ regions the temperature-sensitive lines such as [OIII] $\lambda 4363$ are often too weak to be detected. For such $\mathrm{H}_{\text {II }}$ regions, the relation between strong oxygen line intensities and oxygen abundances is used for the abundance determination. The early calibrations were one-dimensional (Pagel et al. 1979; Edmunds \& Pagel 1984; McCall et al. 1985; Dopita \& Evans 1986; Zaritsky et al. $1994)$, i.e. a relation of the type $\mathrm{O} / \mathrm{H}=f\left(R_{23}\right)$ was used, with

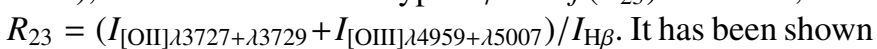
(Pilyugin 2000; 2001a,b) that the oxygen abundances derived with the one-dimensional calibrations are affected by a systematic error. The origin of this systematic error is evident. In a general case, the intensity of oxygen emission lines in $\mathrm{H}$ II regions depends not only on the oxygen abundance but also on the physical conditions (hardness of the ionizing radiation and geometrical factors). Thus, the physical conditions in $\mathrm{H}$ II regions (e.g. via the electronic temperature in the $T_{\mathrm{e}}$-method) should be taken into account to derive accurate oxygen abundances from emission line intensities. In one-dimensional calibrations the physical conditions in $\mathrm{H}$ II regions are ignored. Starting from the idea of McGaugh (1991) that the strong oxygen lines contain the information needed to determine accurate abundances in $\mathrm{H}$ II regions, it has been shown that the physical conditions in $\mathrm{H}_{\text {II }}$ regions can be estimated and taken into account via the excitation parameter $P$ (see Fig. 1). A twodimensional or parametric calibration (the $P$-method) has been proposed (Pilyugin 2000, 2001a,c). A more general relation of the type $\mathrm{O} / \mathrm{H}=f\left(P, R_{23}\right)$ is used in the $P$-method, compared to the relation of the type $\mathrm{O} / \mathrm{H}=f\left(R_{23}\right)$ used in one-dimensional

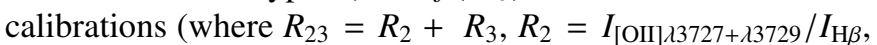

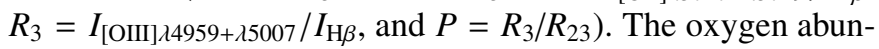
dances derived via the $P$-method are in agreement with the abundances determined using the $T_{\mathrm{e}}$-method over the full range of abundances (see Fig. 1, and Melbourne et al. 2004). This allows us to conclude that the $P$-method provides reliable abundance determinations in $\mathrm{H}$ II regions. 
The correlations between oxygen abundance traced by the individual $\mathrm{H}_{\mathrm{II}}$ regions and macroscopic properties of spiral galaxies have been investigated by numerous authors (Garnett \& Shields 1987; Vila-Costas \& Edmunds 1992; Zaritsky et al. 1994; Garnett 2002; among others). The early one-dimensional $R_{23}$ calibrations were used in these studies for the oxygen abundance determination. Therefore, the results of these studies are not beyond question and must be revised. A compilation of published spectra of $\mathrm{H}$ II regions in spiral galaxies has been carried out in our previous paper (Pilyugin et al. 2004, hereafter Paper I). The oxygen and nitrogen abundances in all the $\mathrm{H}_{\mathrm{II}}$ regions were determined in the same way, using the $P$-method. The correlations between oxygen abundance and macroscopic properties of spiral galaxies have been investigated.

In a pioneering study, Kennicutt (1992) published the global, i.e. spatialy unresolved, spectrophotometry of a sample of 90 galaxies spanning the entire Hubble sequence. Kobulnicky et al. (1999) have shown that the oxygen abundances determined through the $R_{23}$-method of Pagel et al. (1979) using the global emission-line spectra are in agreement with oxygen abundances measured at 0.4 isophotal radius using individual $\mathrm{H}_{\mathrm{II}}$ regions. They concluded that the global emission-line spectra can reliably indicate the chemical properties of galaxies. Based on this conclusion, Melbourne \& Salzer (2002) have estimated the oxygen abundance for a large sample of 519 star-forming emission-line galaxies from the KPNO International Spectroscopic Survey (KISS) and derived the luminosity-metallicity $(L-Z)$ relation. During the last few years, chemical abundances have been derived from the global spectra of a large sample of early-type (e.g. Wegner et al. 2003) and star-forming galaxies (Melbourne \& Salzer 2002; Contini et al. 2002; Tremonti et al. 2003; Lamareille et al. 2004). Contini et al. (2002) have determined the chemical abundances in a sample of UV-selected local and intermediateredshift galaxies from their global spectra. The N/O-O/H diagram obtained by Contini et al. (2002) for these galaxies is not in agreement with the one derived for individual $\mathrm{H}$ II regions in normal spiral and irregular galaxies (e.g. Pilyugin et al. 2003). For a given metallicity, a significant number of the UV-selected galaxies show lower N/O abundance ratios as compared to the individual $\mathrm{H}$ II regions.

The $\mathrm{N} / \mathrm{O}-\mathrm{O} / \mathrm{H}$ diagram is a key relation for understanding the origin of nitrogen and the star formation history of galaxies. Therefore it is very important to understand the origin of the low $\mathrm{N} / \mathrm{O}$ ratios derived in a large fraction of UV-selected galaxies. It has been suggested (Contini et al. 2002; Mouhcine \& Contini 2002) that the low N/O abundance ratios in UV-selected galaxies are due to the fact that UV-selected galaxies are observed at a special stage of their evolution. They have just undergone a powerful starburst which enriched their interstellar medium in oxygen. In these objects nitrogen may not have been completely released.

It is possible, however, that the low N/O ratios in $\mathrm{UV}$-selected galaxies are caused by systematic errors in the determination of N/O abundance ratios. Indeed, the abundances in UV-selected galaxies were determined from the global spectra using the calibration of Kobulnicky et al. (1999, see Fig. 1a). This calibration is based on the grid of $\mathrm{H}$ II region models
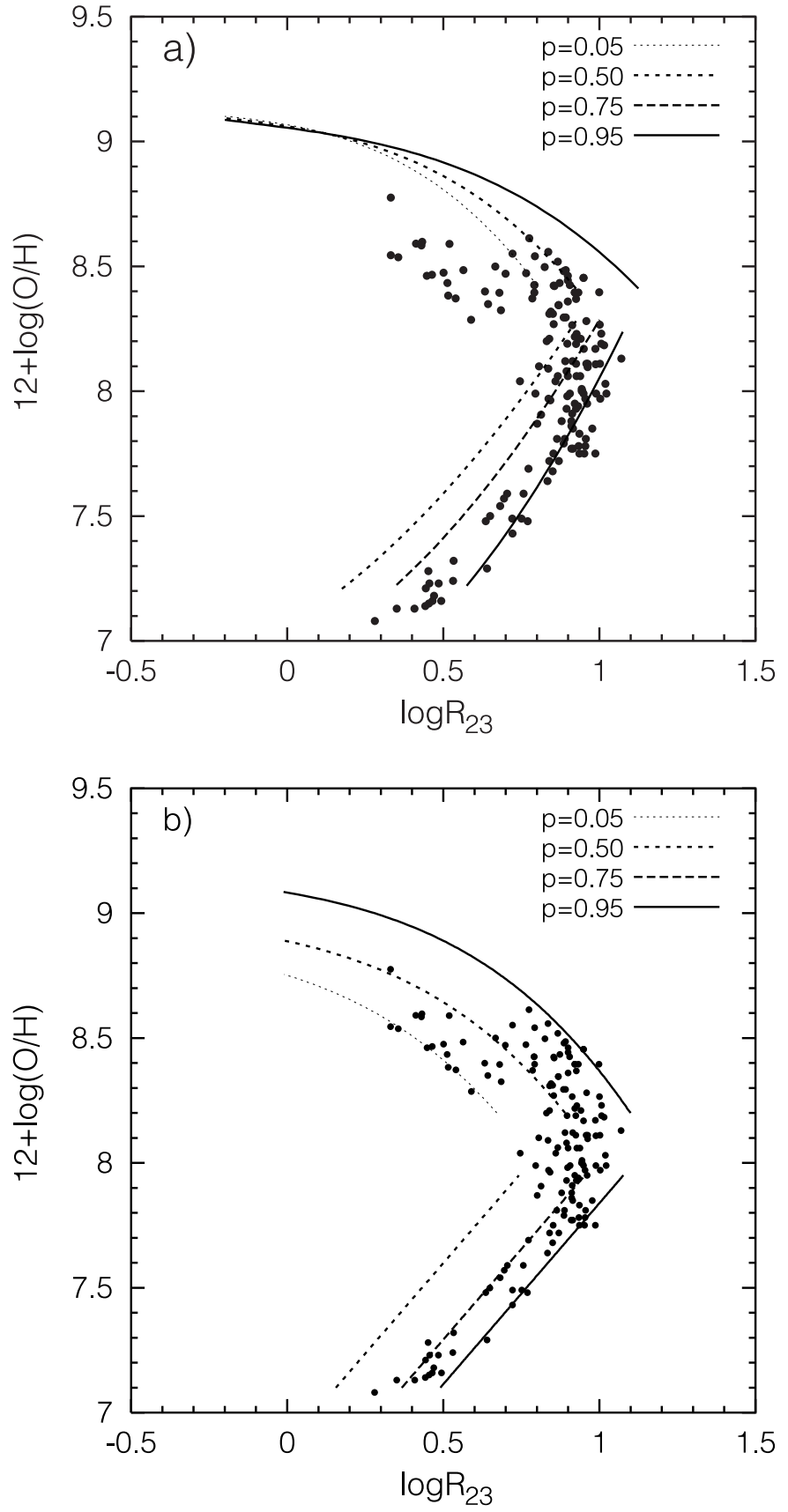

Fig. 1. The "strong line - oxygen abundance" calibrations. a) Points correspond to $\mathrm{H}$ II regions with oxygen abundances determined using direct measurements of $T_{\mathrm{e}}$-method (from the compilation of Pilyugin 2000, 2001a). The lines show the predictions of the two-dimensional theoretical (model) calibration of Kobulnicky et al. (1999) parameterized for different values of the excitation parameter $P$. Every line is labeled with the corresponding value of the excitation parameter $P$. b) The lines are the predictions of the two-dimensional empirical calibrations obtained for the lower branch $(12+\log (\mathrm{O} / \mathrm{H})<8.0$; Pilyugin 2000, 2001c) and for the upper branch $(12+\log (\mathrm{O} / \mathrm{H})>8.2$; Pilyugin 2001a). Each $\mathrm{O} / \mathrm{H}-R_{23}$ relation is labeled with the corresponding value of the excitation parameter $P$. The points are the same as in panel a).

of McGaugh (1991). The N/O-O/H diagram of Pilyugin et al. (2003) is based on the abundances derived in individual 
H II regions using the $P$-method (or via the classical $T_{\mathrm{e}}$-method when possible). It should be stressed that "strong lines - oxygen abundance" calibrations do not form an uniform family. One should clearly recognize that there are two different types of calibrations. The calibrations of the first type are empirical, i.e. based on oxygen abundances in $\mathrm{H}_{\text {II }}$ regions determined via the $T_{\mathrm{e}}$-method. The calibrations of Pilyugin are twodimensional calibrations of this type. The calibrations of the second type are "theoretical" (or model) calibrations, i.e. based on the grid of photoionization models of $\mathrm{H}$ II regions. The calibration of Kobulnicky et al. (1999) is the two-dimensional calibration of this type.

Inspection of Fig. 1 shows that the discrepancy between the calibration of Kobulnicky et al. (1999) and the calibrations of Pilyugin is very small for the very low-metallicity $(12+$ $\log (\mathrm{O} / \mathrm{H}) \sim 7.3)$, high-excitation $\mathrm{H}$ in regions. But the discrepancy increases with increasing metallicity and with decreasing excitation parameter, reaching the value of $\Delta \log (\mathrm{O} / \mathrm{H}) \sim$ 0.15 dex for $\mathrm{H}_{\text {II }}$ regions with $12+\log (\mathrm{O} / \mathrm{H}) \sim 7.9$. The agreement between the calibrations of Kobulnicky et al. (1999) and those of Pilyugin disappears for high-metallicity $\mathrm{H}_{\text {II }}$ regions that lie on the upper branch of the $\mathrm{O} / \mathrm{H}-R_{23}$ relation (see Pilyugin 2003 for a more detailled discussion). The calibrations of Kobulnicky et al. (1999) do not reproduce the $T_{\mathrm{e}}$-based abundances for high-metallicity $\mathrm{H}$ in regions that lie on the upper branch of the $\mathrm{O} / \mathrm{H}-R_{23}$ relation. Then, it is possible that the low N/O ratios in UV-selected galaxies is caused by systematic errors in the determination of abundances. Therefore, to establish whether the low N/O abundance ratios of UV-selected galaxies are real or artificial, it is necessary to derive abundances for UV-selected galaxies through the $P$-method. This is one of the main goals of this work.

The plan of the paper is the following. In the next section, the validity of the abundances derived from the global spectra through the $P$-method is examined. The $\mathrm{N} / \mathrm{O}-\mathrm{O} / \mathrm{H}$ diagram for UV-selected galaxies is built and compared with the data from Pilyugin et al. (2003) in Sect. 3. In Sect. 4 the luminosity-metallicity relation for the UV-selected galaxies is derived and compared with samples of normal nearby spiral galaxies (Jansen et al. 2000; Paper I) and with the luminositymetallicity relation obtained for intermediate-redshift galaxies. Section 5 is a brief conclusion.

\section{On the validity of abundances derived from global spectra of galaxies}

The validity of abundances derived from the global spectrum of galaxies, using the $P$-method, will be examined in the following way. A model spectrum for a galactic disk is built using individual spectra of $\mathrm{H}_{\text {II }}$ regions. The disk is divided into $n$ rings. The global spectrum of such an "artificial" galaxy is built by summing the spectra of $n$ rings. For the sake of clarity, the spectrum of an individual ring is represented by the spectrum of a single $\mathrm{H}$ II region. The compilation of published line intensities for a large sample of individual $\mathrm{H}$ II regions in spiral and irregular galaxies (Paper I; Pilyugin et al. 2003) has been used to perform this work. The $\mathrm{N} / \mathrm{O}$ versus $\mathrm{O} / \mathrm{H}$ diagram for our collection of $\mathrm{H}_{\mathrm{II}}$ regions is shown in Fig. 2. We select the basic

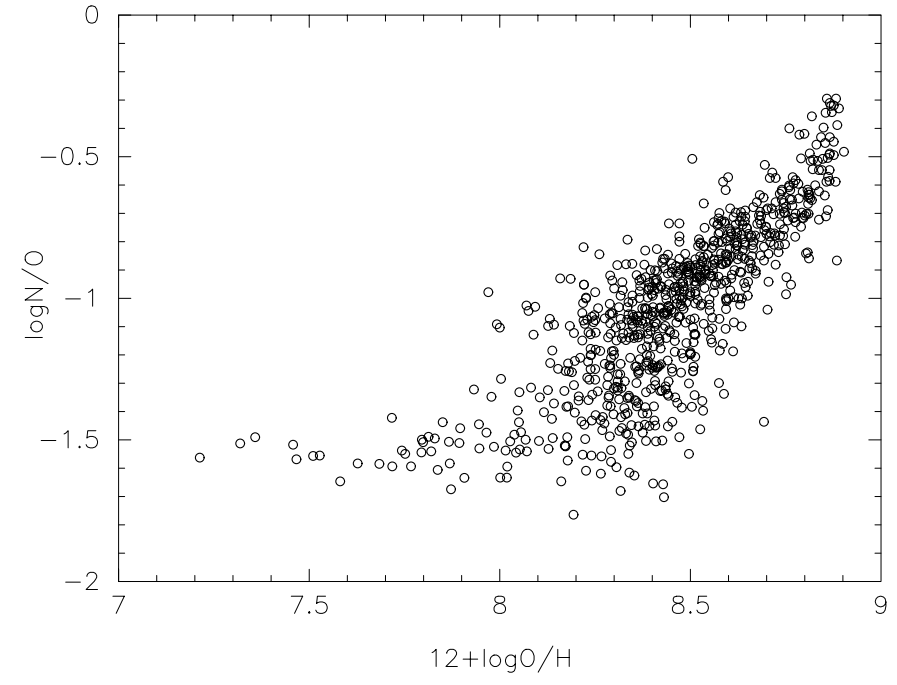

Fig. 2. The $\mathrm{N} / \mathrm{O}$ vs. $\mathrm{O} / \mathrm{H}$ diagram for our collection of $\mathrm{H}$ II regions in spiral and irregular galaxies.

subsample of $\mathrm{H}$ II regions in such a way that this subsample reproduces a sequence with a more or less monotonic decrease of oxygen and nitrogen abundances (with a step $\Delta \log (\mathrm{O} / \mathrm{H}) \sim$ 0.02 dex) and reflects the general behaviour of the nitrogen-tooxygen ratio (Fig. 3). This subsample of $\mathrm{H}$ II regions has been used to build the basic sequence of model galaxy spectra. The large scatter observed in the $\mathrm{N} / \mathrm{O}$ vs. $\mathrm{O} / \mathrm{H}$ diagram (Fig. 2) is due to the fact that data for spiral galaxies of different morphological types are presented. Early-type spirals (Sa, Sab) show high N/O ratios whereas late-type spirals ( $\mathrm{Sd}, \mathrm{Sdm}$ ) have a lower N/O ratio at a given $\mathrm{O} / \mathrm{H}$ (Pilyugin et al. 2003). The basic subsample of selected $\mathrm{H}$ in regions is close to the lower envelope in the N/O vs. $\mathrm{O} / \mathrm{H}$ diagram. This subsample thus corresponds to spiral galaxies of late morphological types. Galaxy model spectra with other subsamples of $\mathrm{H}$ II regions have been also constructed.

The oxygen abundance in the disk of spiral galaxies can be specified by the maximum (or central intersect oxygen abundance), $Z_{\max }=12+\log (\mathrm{O} / \mathrm{H})($ at $r=0)$, and the minimum oxygen abundance (or oxygen abundance at the isophotal radius), $Z_{\min }=12+\log (\mathrm{O} / \mathrm{H})\left(\right.$ at $\left.r=R_{25}\right)$. The radial oxygen abundance distributions in the disk of 53 well-studied spiral galaxies have been determined in Paper I using the abundances in individual $\mathrm{H}$ II regions derived via the $P$-method. The extrapolated central intersect values of $\mathrm{O} / \mathrm{H}$ and oxygen abundances at the isophotal radius $R_{25}$ for these galaxies are presented in Fig. 4. The galaxies are plotted in decreasing central $\mathrm{O} / \mathrm{H}$ order. Figure 4 shows that both the maximum and the minimum oxygen abundances can significantly vary from galaxy to galaxy. Therefore we have built a basic sequence of galaxy model spectra with different central intersect oxygen abundances $Z_{\max }=12+\log (\mathrm{O} / \mathrm{H})($ at $r=0)$ and a fixed oxygen abundance at the isophotal radius, $Z_{\min }=12+\log (\mathrm{O} / \mathrm{H})$ (at $r=R_{25}$ ) 8.1. It should be emphazised that the goal of the present section is to test the credibility of abundances derived from global spectra via the $P$-method but not to reproduce all the galaxies from Paper I. The value of $Z_{\min }$ was choosen in a 

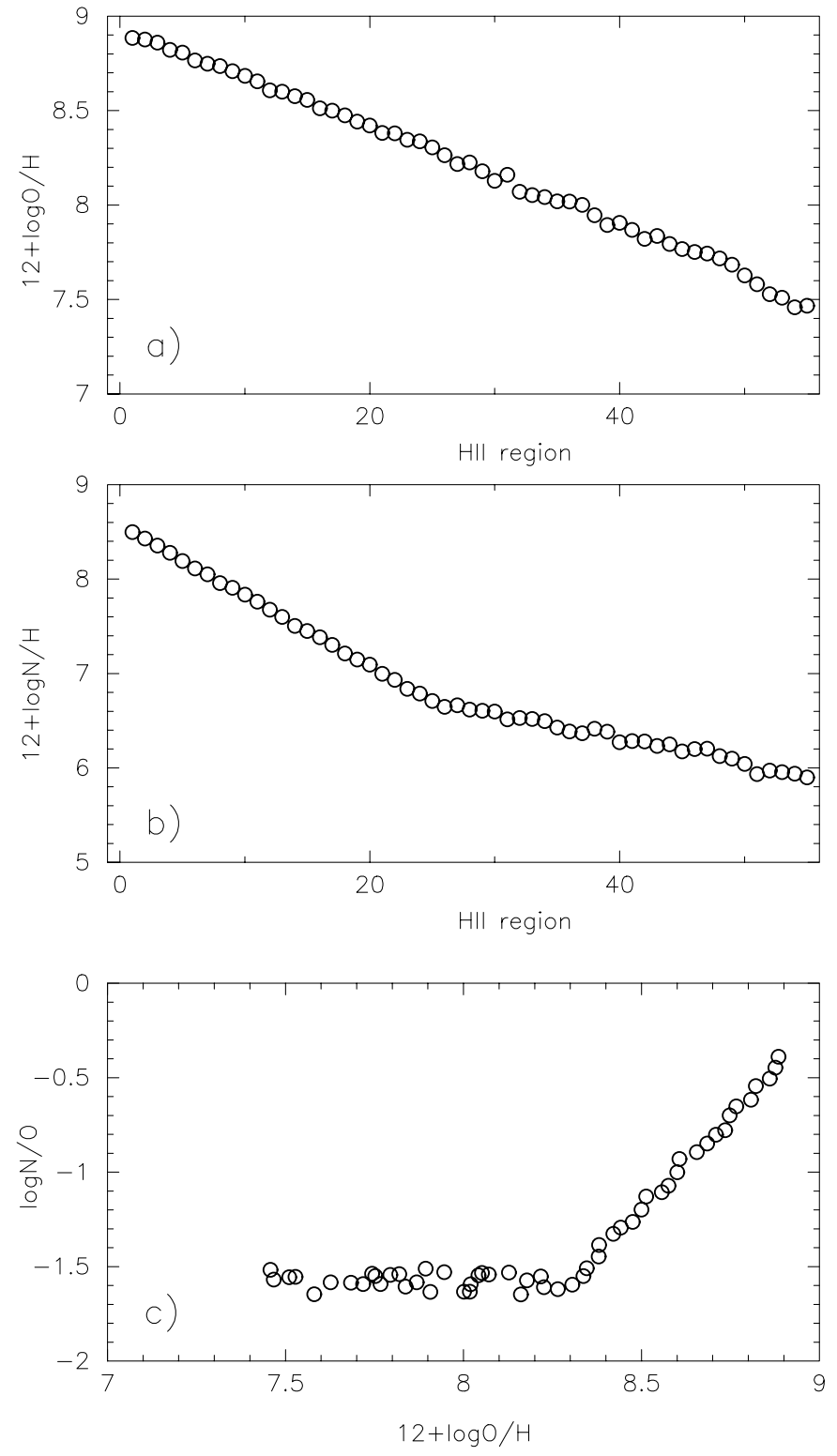

Fig. 3. Oxygen a) and nitrogen b) abundances as well as the N/O-O/H diagram c) for a basic subset of $\mathrm{H}_{\text {II }}$ regions selected for the construction of an artificial galaxy.

such way that the basic sequence of model spectra reproduces a similar fraction of galaxies in which the bulk of $\mathrm{H}$ II regions belong to the upper branch of the $\mathrm{O} / \mathrm{H}-R_{23}$ diagram (at high values of $\Delta Z=Z_{\max }-Z_{\min }$ ) and galaxies in which a significant number of $\mathrm{H}$ II regions belong to the lower branch of the $\mathrm{O} / \mathrm{H}-R_{23}$ diagram (at low values of $\Delta Z=Z_{\max }-Z_{\min }$ ). Other sequences of galaxy model spectra (with other values of $Z_{\min }$, and/or with other subsamples of $\mathrm{H}$ II regions) have been also constructed. It has been found that the results obtained for other sequences of models do not differ from the ones obtained with the basic sequence of models. Therefore, we will only report the results obtained with the basic sequence of models.

Using the line fluxes $I_{j}^{\lambda}$ for individual $H_{\text {II }}$ regions normalized to $I_{j}^{\mathrm{H} \beta}$, the global line fluxes $I_{\mathrm{g}}^{\lambda}$ normalized to $I_{\mathrm{g}}^{\mathrm{H} \beta}$

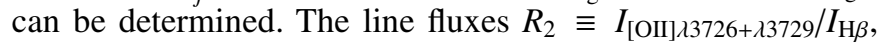

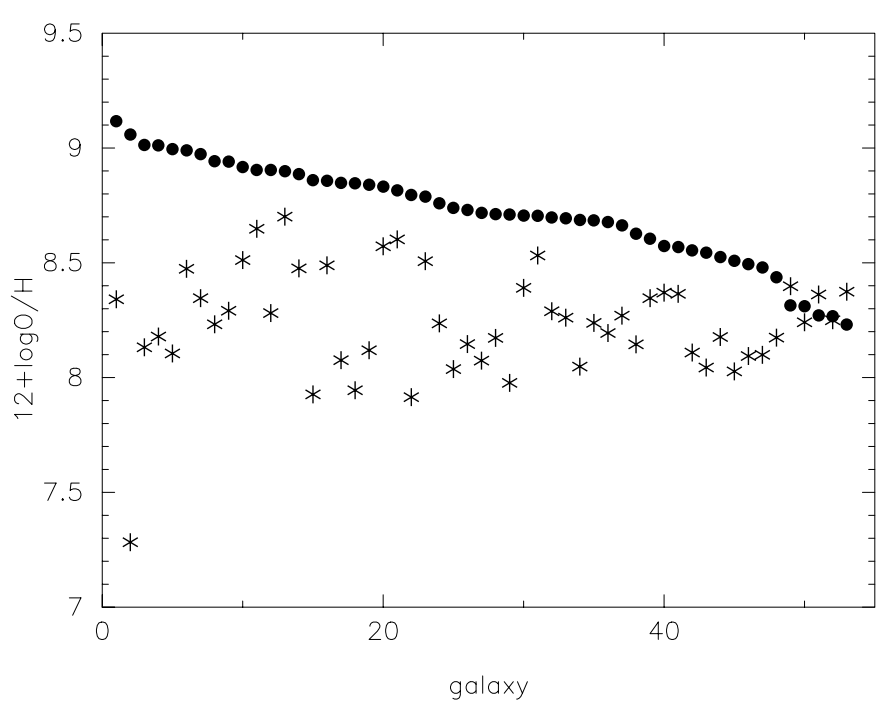

Fig. 4. Central intersect oxygen abundances (filled circles) and oxygen abundances at isophotal radius (asterisks) for the set of spiral galaxies from Paper I. The galaxies are plotted in decreasing central $\mathrm{O} / \mathrm{H}$ order.

$R_{3} \equiv I_{[\mathrm{OIII}] \lambda 4959+\lambda 5007} / I_{\mathrm{H} \beta}$, and $N_{2} \equiv I_{[\mathrm{NII}] \lambda 6548+\lambda 6584} / I_{\mathrm{H} \beta}$ are used in the present study. The radial distribution of the number density of $\mathrm{H}_{\text {II }}$ regions changes significantly from galaxy to galaxy (Rozas et al. 1996). The radial flux density distribution of all the $\mathrm{H}$ II regions is very irregular. These variations of the flux density are stronger than the systematic variations of the flux density with galactocentric distance (Rozas et al. 1999). Therefore the global oxygen and nitrogen line fluxes $I_{\mathrm{g}}^{*, \lambda}$ were derived as:

$I_{\mathrm{g}}^{*, \lambda}=\sum_{j=1}^{n} w_{j} I_{j}^{\lambda}$,

and the global $\mathrm{H}_{\beta}$ line flux $I_{\mathrm{g}}^{*, \mathrm{H}_{\beta}}$ was derived as

$I_{\mathrm{g}}^{*, \mathrm{H}_{\beta}}=\sum_{j=1}^{n} w_{j}$,

where $n$ is the number of rings in a given model, and $\mathrm{w}_{j}$ are random numbers in the range $0 \div 1$. At a given $j$ the value $\mathrm{w}_{j}$ is the same in Eqs. (1) and (2). We would like to stress that the random distribution is not adopted for the luminosity function of $\mathrm{H}$ II regions but for the radial flux distribution of all the $\mathrm{H}$ II regions. The number of rings $n$ in a given model is defined by the value $\Delta Z=Z_{\max }-Z_{\min }$ and corresponds to the number of $H_{\text {II }}$ regions with metallicity between $Z_{\min }$ and $Z_{\max }$ in the selected subsample. Then the global line fluxes $I_{\mathrm{g}}^{\lambda}$ normalized to $I_{\mathrm{g}}^{\mathrm{H} \beta}$ are determined as:

$I_{\mathrm{g}}^{\lambda}=\frac{\sum_{j=1}^{n} w_{j} I_{j}^{\lambda}}{\sum_{j=1}^{n} w_{j}}$.

The global line fluxes derived from Eq. (3) are used for the determination of global values of oxygen and nitrogen abundances. The global oxygen and nitrogen abundances are derived within a two-zone model for the temperature structure in the same way as in Paper I and Pilyugin et al. (2003). As a 


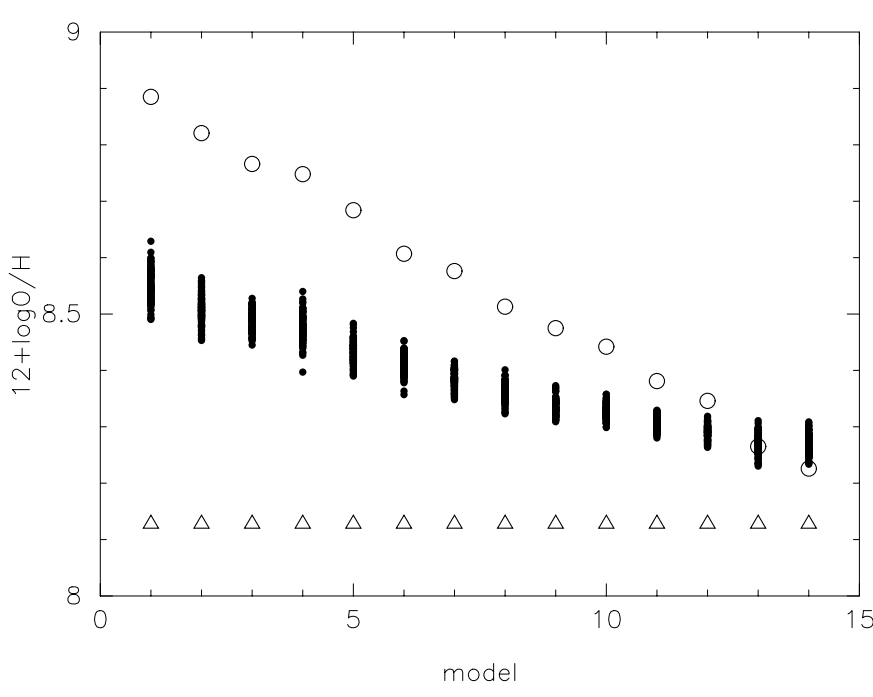

Fig. 5. The maximum ("central intersect") oxygen abundance (large open circles), the minimum ("at the isophotal radius") oxygen abundance (open triangles), and the oxygen abundance derived from the global spectra (filled circles) for models of the basic serie.

first step, the $(\mathrm{O} / \mathrm{H})_{P}$ oxygen abundance is determined with the expression suggested in Pilyugin (2001):

$12+\log (\mathrm{O} / \mathrm{H})_{P}=\frac{R_{23}+54.2+59.45 P+7.31 P^{2}}{6.07+6.71 P+0.37 P^{2}+0.243 R_{23}}$,

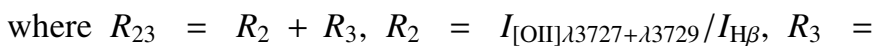

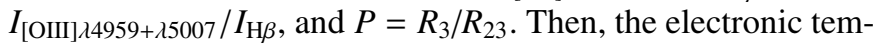
peratures $T_{\mathrm{e}}([\mathrm{OII}])$ and $T_{\mathrm{e}}([\mathrm{OIII}])$ are derived using the value of $\mathrm{O} / \mathrm{H}$ derived from Eq. (4) and emission line fluxes derived from Eq. (3). For this purpose the expressions for oxygen abundance determination from Pagel et al. (1992) and the $T_{\mathrm{e}}([\mathrm{OII}])-T_{\mathrm{e}}([\mathrm{OIII}])$ relation from Garnett (1992) are used. Then, assuming $t_{[\mathrm{NII}]}=t_{[\mathrm{OII}]}$, the $\mathrm{N} / \mathrm{O}$ abundance ratio is determined from the expression given in Pagel et al. (1992).

The $20 \div 100$ variants of random distributions of $\mathrm{H}$ II region $\mathrm{H}_{\beta}$ luminosities have been considered for every model of galaxy with fixed values of $Z_{\min }$ and $Z_{\max }$. The oxygen abundances derived from the global spectra of the basic sequence of models are presented in Fig. 5 (filled circles) together with $Z_{\max }$ (open circles) and $Z_{\min }$ (open triangles) values. The mean value of global oxygen abundances from a series of basic models, obtained by averaging the individual global oxygen abundances for different variants of random distributions of $\mathrm{H}_{\text {II }}$ region $\mathrm{H}_{\beta}$ luminosities, versus oxygen abundance at galactocentric distances $r=0.3 R_{25}$ (asterisks), $r=0.4 R_{25}$ (open circles), and $r=0.5 R_{25}$ (pluses) are presented in Fig. 6. The oxygen abundance at a given galactocentric distance is determined here as $12+\log (\mathrm{O} / \mathrm{H})_{r=c R_{25}}=12+\log (\mathrm{O} / \mathrm{H})_{\max }-$ $\left.c\left[\log (\mathrm{O} / \mathrm{H})_{\max }-\log (\mathrm{O} / \mathrm{H})_{\min }\right]\right)$, where $c=0.3,0.4$, and 0.5 . The data for the sequence of models with $Z_{\min }=8.42$ are also presented in Fig. 6 by the filled circles. The data for the sequence $\mathrm{B}$ of models (based on other subsample of $\mathrm{H}$ II regions, see below) with $Z_{\min }=8.30$ are presented in Fig. 6 by the open squares. Examination of Figs. 5 and 6 shows that the oxygen abundance derived from the global emission-line spectrum via the $P$-method agree (within $0.1 \mathrm{dex}$ ) with the oxygen

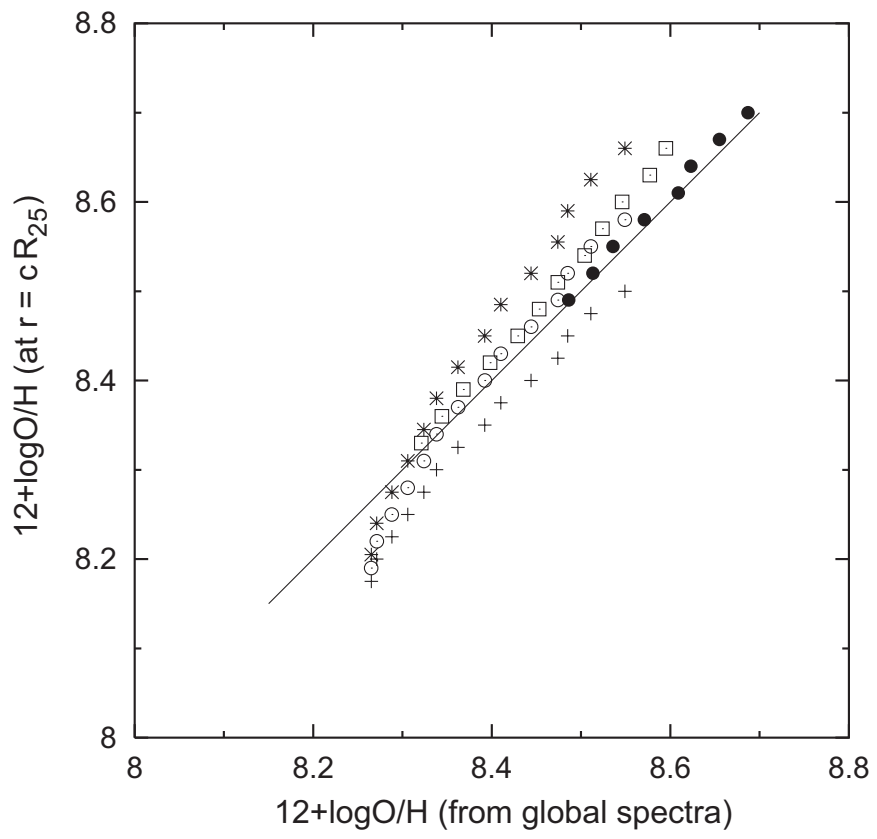

Fig. 6. Oxygen abundances derived from the global spectra versus oxygen abundances at $r=c \times R_{25}$. The pluses correspond to abundances at $r=0.5 \times R_{25}$ for basic series of models with $Z_{\min }=8.12$. The open circles corresponds to abundance at $r=0.4 \times R_{25}$ for basic series of models with $Z_{\min }=8.12$. The asterisks correspond to abundances at $r=0.3 \times R_{25}$ for basic series of models with $Z_{\min }=8.12$. The filled circles correspond to abundances at $r=0.4 \times R_{25}$ for basic series of models with $Z_{\min }=8.42$. The open squares correspond to abundances at $r=0.4 \times R_{25}$ for series $\mathrm{B}$ of models with $Z_{\min }=8.30$.

abundance at galactocentric distance $r=0.4 R_{25}$, traced by individual $\mathrm{H}_{\text {II }}$ regions, if most of the $\mathrm{H}_{\text {II }}$ regions belong to the upper branch of the $\mathrm{O} / \mathrm{H}-R_{23}$ relation. It is worth noting that the oxygen abundances at galactocentric distance $r=0.4 R_{25}$ for the series B of the models are slightly higher than the global oxygen abundances. The basic subsample of $\mathrm{H}$ II regions corresponds to late-type spiral galaxies. The subsample B of $\mathrm{H}_{\text {II }}$ regions are close to the upper envelope in the N/O versus $\mathrm{O} / \mathrm{H}$ diagram (Fig. 7) and corresponds to early-type spiral galaxies. Then, the global oxygen abundances derived for models of late-type galaxies are rather close to oxygen abundances at galactocentric distance $r=0.4 R_{25}$ while the values derived for models of early-type galaxies are slightly lower. The disagreement between the global oxygen abundances and the oxygen abundances at galactocentric distance $r=0.4 R_{25}$ for all the models does not exceed 0.1 dex. This is in agreement with the conclusion of Kobulnicky et al. (1999) that global emission-line spectra can reliably indicate the chemical properties of galaxies.

The N/O ratios derived from global spectra of the basic serie of models are presented in Fig. 7 (points) as a function of the global oxygen abundance, together with the positions of individual $\mathrm{H}_{\text {II }}$ regions (open circles) from the basic subsample. The N/O ratios derived from global spectra of the serie B of models are presented by the filled squares, the corresponding subsample of individual $\mathrm{H}_{\text {II }}$ regions are shown by plus symbols. Figure 7 shows that the N/O ratios derived from global 


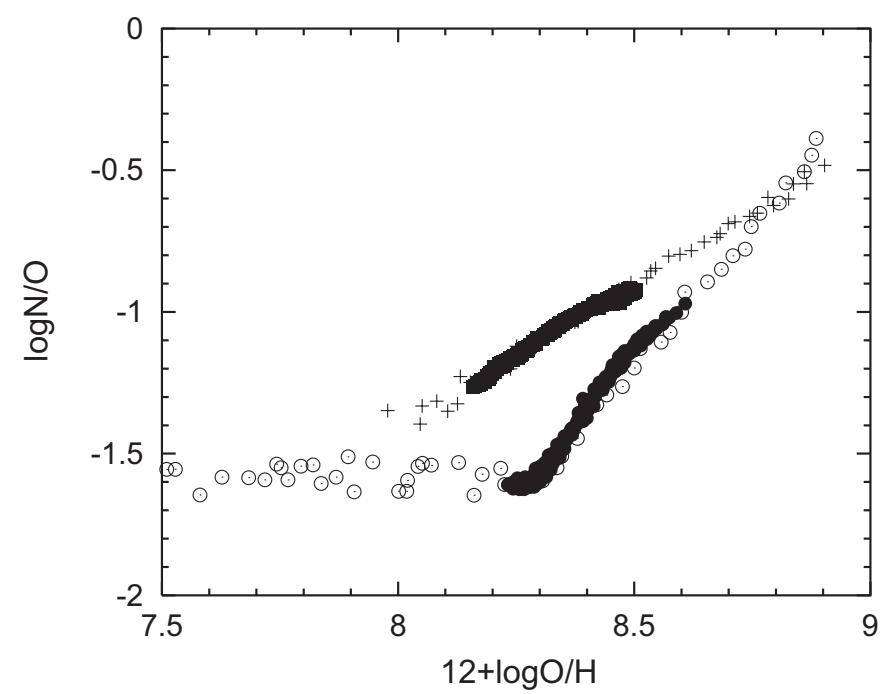

Fig. 7. N/O vs. O/H diagram. The filled circles are the global abundances derived for the models of the basic serie from Fig. 5, the open circles are the corresponding selected subsets of individual $\mathrm{H}_{\text {II }}$ regions. The filled squares are the global abundances derived for the models of serie B with $Z_{\min }=8.1$, the pluses are the corresponding selected subsets of individual $\mathrm{H}$ II regions.

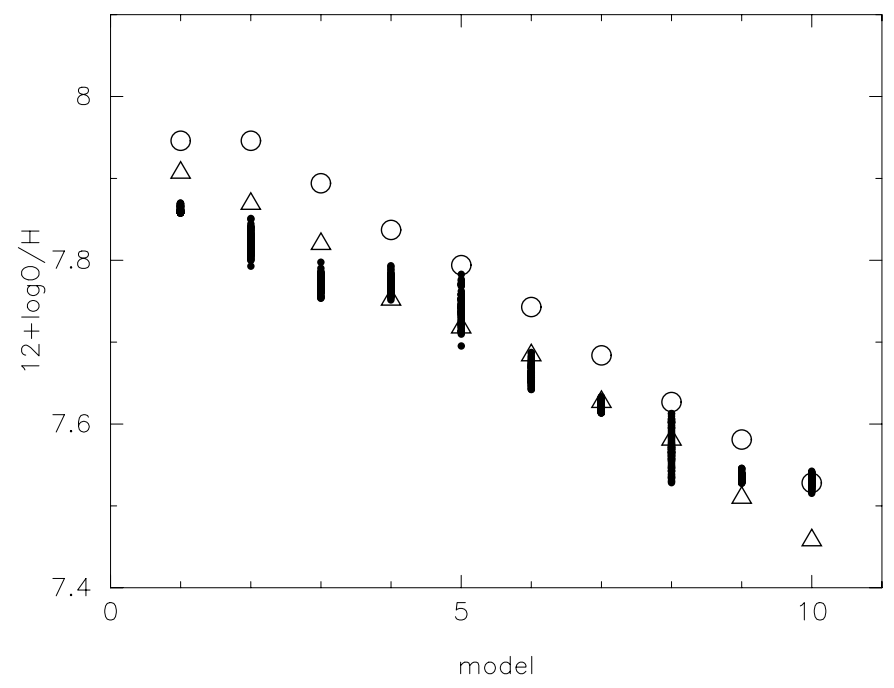

Fig. 8. The maximum ("central intersect") oxygen abundance (large open circles), the minimum ("at the isophotal radius") oxygen abundance (open triangles), and the oxygen abundance derived from the global spectra (filled circles) for models of low-metallicity galaxies.

spectra lie in the same region of the $\mathrm{N} / \mathrm{O}-\mathrm{O} / \mathrm{H}$ diagram as the individual $\mathrm{H}$ II regions populating these galaxies.

The models of high-metallicity galaxies, i.e. models where all or the majority of $\mathrm{H}_{\mathrm{II}}$ regions are metal-rich $(12+$ $\log (\mathrm{O} / \mathrm{H})>8.2)$ and lie on the upper branch of the $\mathrm{O} / \mathrm{H}-R_{23}$ diagram, have been considered above. Now model spectra for the low-metallicity galaxies, populated by metalpoor $\mathrm{H}$ II regions $(12+\log (\mathrm{O} / \mathrm{H})<8.2)$ from the lower branch of the $\mathrm{O} / \mathrm{H}-R_{23}$ diagram, will be considered. It is well known that the low-metallicity (irregular) galaxies show no significant radial abundance gradient. Therefore the value of $\Delta Z=$ $Z_{\max }-Z_{\min } \sim 0.1$ dex is adopted. The $\mathrm{H}$ II regions from the basic

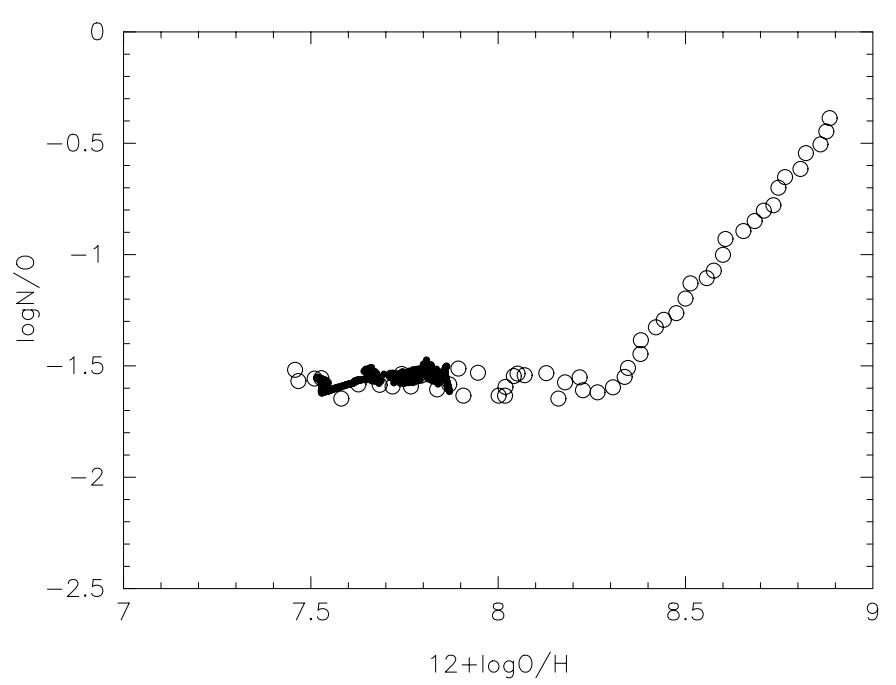

Fig. 9. The N/O vs. O/H diagram. The filled circles are the global abundances derived for the models of low-metallicity galaxies from Fig. 8. The open circles are the selected subsets of individual $\mathrm{H}$ II regions.

subsample are used. Again the 100 variants of random distributions of $\mathrm{H}$ II region $\mathrm{H}_{\beta}$ luminosities have been considered for each "artificial" galaxy with given $Z_{\min }$ and $Z_{\max }$. The corresponding relation for low-metallicity $\mathrm{H}_{\text {II }}$ regions (Pilyugin 2001a,c) has been used for the oxygen abundance determination instead of Eq. (4). The oxygen abundances in lowmetallicity galaxies derived from the global spectra are presented in Fig. 8 (points) together with the $Z_{\max }$ (open circles) and $Z_{\min }$ (open triangles) values. Inspection of Fig. 8 shows that the oxygen abundances derived from the global emissionline spectra of a galaxy through the $P$-method are slightly lower than the oxygen abundances in individual H II regions. It should be noted that the same effect has been revealed for the $T_{\mathrm{e}}$-method by Kobulnicky et al. (1999). They have found that the oxygen abundances derived from the global emissionline spectra of low-metallicity galaxies via the $T_{\mathrm{e}}$-method are slightly underestimated, by $\Delta(\mathrm{O} / \mathrm{H}) \leq 0.1$ dex.

The N/O ratios in low-metallicity galaxies derived from global spectra are presented in Fig. 9 (points) as a function of the oxygen abundance, together with the positions of individual $\mathrm{H}$ iI regions (open circles) from the basic subsample. Figure 9 shows that the N/O ratios derived from global spectra of lowmetallicity galaxies occupy the same band in the $\mathrm{N} / \mathrm{O}-\mathrm{O} / \mathrm{H}$ diagram as the individual $\mathrm{H}$ II regions.

These results show that the oxygen abundances derived from the global emission-line spectra of high-metallicity galaxies via the $P$-method agree within 0.1 dex with the oxygen abundances at $r=0.4 R_{25}$, traced by individual $\mathrm{H}_{\text {II }}$ regions, if most of $\mathrm{H}$ II regions belong to the upper branch of the $\mathrm{O} / \mathrm{H}-\mathrm{R}_{23}$ relation. The oxygen abundances in low-metallicity galaxies derived from the global emission-line spectra of galaxies via the $P$-method are slightly underestimated by $\Delta(\mathrm{O} / \mathrm{H}) \leq$ 0.1 dex. The N/O ratios in high- and low-metallicity galaxies derived from global spectra occupy the same band in the $\mathrm{N} / \mathrm{O}-\mathrm{O} / \mathrm{H}$ diagram as the individual $\mathrm{H}$ in regions. Our results confirm the conclusion of Kobulnicky et al. (1999) that the 
global emission-line spectra can reliably indicate the chemical properties of galaxies.

\section{The chemical abundances in UV-selected galaxies}

We have shown that the $P$-method can be used to derive chemical abundances from the global emission-line spectra of galaxies. As in case of individual $\mathrm{H}$ II regions, one has to know apriori on which of the two branches the galaxy lies. We will try to solve this problem with the following strategy. We will compare the global spectrum of a galaxy with the spectrum of a large number of individual $\mathrm{H}_{\text {II }}$ regions with known oxygen abundances. The oxygen abundance of a $\mathrm{H}$ II region, which shows the minimum spectral difference with the global spectrum of a galaxy, can be adopted as the global oxygen abundance of this galaxy. The difference between the global spectrum of a galaxy and the spectrum of an individual $\mathrm{H}$ II region can be specified by the parameter $D$ :

$$
\begin{aligned}
D= & {\left[\left(\log R_{2}^{\mathrm{g}}-\log R_{2}^{\mathrm{HII}}\right)^{2}+\left(\log R_{3}^{\mathrm{g}}-\log R_{3}^{\mathrm{HII}}\right)^{2}\right.} \\
& \left.+\left(\log N_{2}^{\mathrm{g}}-\log N_{2}^{\mathrm{HII}}\right)^{2}\right]^{\frac{1}{2}}
\end{aligned}
$$

where $R_{2}^{\mathrm{g}}, R_{3}^{\mathrm{g}}$, and $N_{2}^{\mathrm{g}}$ are the line fluxes in global spectra of galaxies normalized to the $\mathrm{H}_{\beta}$ flux. $R_{2}^{\mathrm{HII}}, R_{3}^{\mathrm{HII}}$, and $N_{2}^{\mathrm{HII}}$ are the line fluxes in spectra of individual $\mathrm{H}_{\text {II }}$ regions normalized to the $\mathrm{H}_{\beta}$ flux. The use of the logarithms instead of the fluxes in the definition of $D$ is due to the following reason. Since the [OII], [OIII], and [NII] line fluxes can be significantly different, the $D$ parameter would be dominated by the strongest emission lines if fluxes were used in the definition.

The comparison of global spectra of $68 \mathrm{UV}$-selected galaxies from Contini et al. (2002) with spectra of individual $\mathrm{H}$ II regions was carried out using our collection of 785 individual H II regions. The 785 values of the difference index $D_{j}$ have been determined from Eq. (5) for each UV-selected galaxy from the sample of Contini et al. (2002). We choose from $D_{j}$ the minimum value of difference index $D_{\min }$, and the oxygen abundance in the galaxy is adopted to be equal to the oxygen abundance in the corresponding $\mathrm{H}$ II region. The oxygen abundance in the galaxy estimated in such a way will be referred to $(\mathrm{O} / \mathrm{H})_{D}$. Then we choose from $D_{j}$ the minimum value of difference index $D_{L}$ when the spectrum of the galaxy is compared to the spectra of $\mathrm{H}_{\text {II }}$ regions with $12+\log (\mathrm{O} / \mathrm{H}) \leq 8.1$, and the minimum value of difference index $D_{U}$ when the spectrum of the galaxy is compared to the spectra of $\mathrm{H}$ II regions with $12+\log (\mathrm{O} / \mathrm{H})>8.1$. It is evident that the value of $D_{\min }$ is equal to the value of $D_{L}$ or to the value of $D_{U}$. Figure 10 shows the value of $D_{U}$ (open circles) and the values of $D_{L}$ (filled circles) as a function of $12+\log (\mathrm{O} / \mathrm{H})_{D}$ for the sample of $\mathrm{UV}$-selected galaxies.

Inspection of Fig. 10 shows that it is possible to find, among the spectra of individual $\mathrm{H}_{\text {II }}$ regions, a spectrum quite similar to the global spectrum of each UV-selected galaxy. It also shows that, for a fraction of UV-selected galaxies, a similar spectrum can be found among the spectra of both highmetallicity and low-metallicity $\mathrm{H}_{\mathrm{II}}$ regions, the values of $D_{U}$

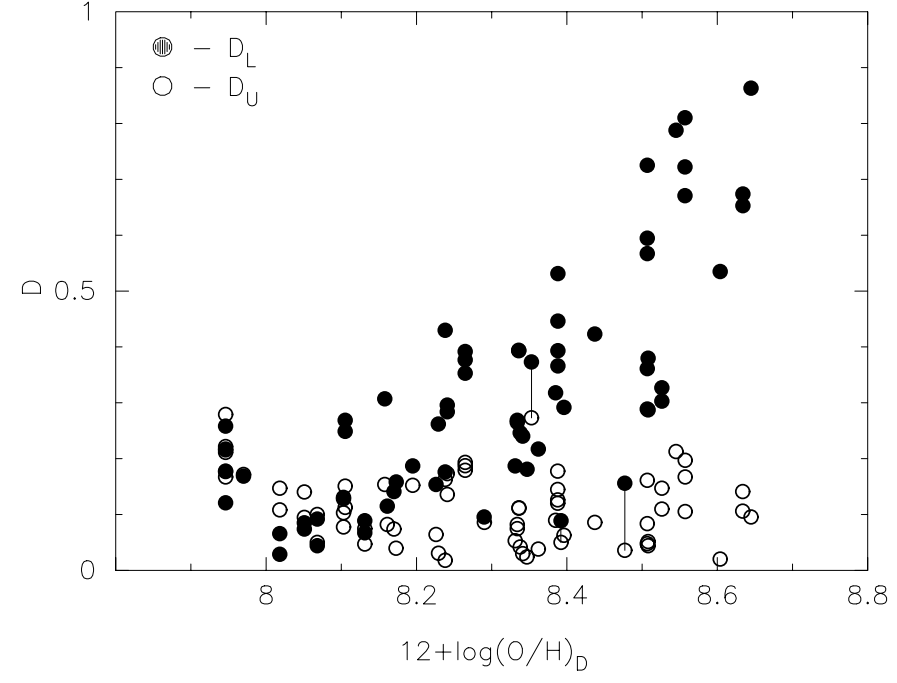

Fig. 10. The difference indexes $D_{U}$ (open circles) and $D_{L}$ (filled circles) as a function of the $12+\log (\mathrm{O} / \mathrm{H})_{D}$ for the sample of UV-selected galaxies (Contini et al. 2002).

and $D_{L}$ being relatively close to each other. But for a significant fraction of UV-selected galaxies, a similar spectrum can be found only among the spectra of high-metallicity $\mathrm{H}_{\text {II }}$ regions, the $D_{U}$ value being significantly lower than the value of $D_{L}$. The value of $D_{U}$ is appeciably lower than the value of $D_{L}$ for galaxies with $12+\log (\mathrm{O} / \mathrm{H})_{D}>8.3$ (Fig. 10). Then, the condition $12+\log (\mathrm{O} / \mathrm{H})_{D}>8.3$ (or $D_{U}<D_{L}$ ) allows us to identify the high-metallicity galaxies. Figure 10 shows that the application of these criteria is not beyond question for three cases. Two galaxies, \#11 and \#47, have low values both for $D_{U}$ and $D_{L}$. On the contrary, both the value of $D_{U}$ and the value of $D_{L}$ are high for the galaxy \#46 from the list of Contini et al. (2002). The values of $D_{U}$ and $D_{L}$ for these galaxies are connected by solid lines in Fig. 10. The values of the difference index $D_{j}$ for the galaxy \#46 ("peculiar" case) versus the oxygen abundances in individual $\mathrm{H}$ II regions are shown in Fig. 11 (panel a). For comparison, the values of the difference index $D_{j}$ for a "normal" case (galaxy \#23) of similar metallicity versus the oxygen abundances in individual $\mathrm{H}_{\mathrm{II}}$ regions are shown in Fig. 11 (panel b). Thus, the condition $12+\log (\mathrm{O} / \mathrm{H})_{D}>8.3$ (or $D_{U}<D_{L}$ ) allows us to identify the high-metallicity galaxies. The confidence level of the identification is defined by the values of $D_{U}$ and $D_{L}$. If the value of $D_{U}$ is low and the value of $D_{L}$ is significantly higher than the value of $D_{U}$ the highmetallicity galaxies are identified with a high probability.

Thus, the comparison of the global spectrum of a galaxy with a collection of spectra of individual $\mathrm{H}_{\text {II }}$ regions can be used to distinguish high- versus low-metallicity objects. To verify this conclusion, the sample of nearby field galaxies from Jansen et al. (2000) has been considered. Figure 12 shows the values of $D_{U}$ (open circles) and $D_{L}$ (filled circles) as a function of $12+\log (\mathrm{O} / \mathrm{H})_{D}$ for the sample of Jansen et al. $(2000)^{1}$. Comparison of Figs. 10 and 12 shows that the trends of values

\footnotetext{
1 Jansen et al. (2000) published both the integrated and nuclear spectra of galaxies. For this study, we use only the integrated spectra.
} 

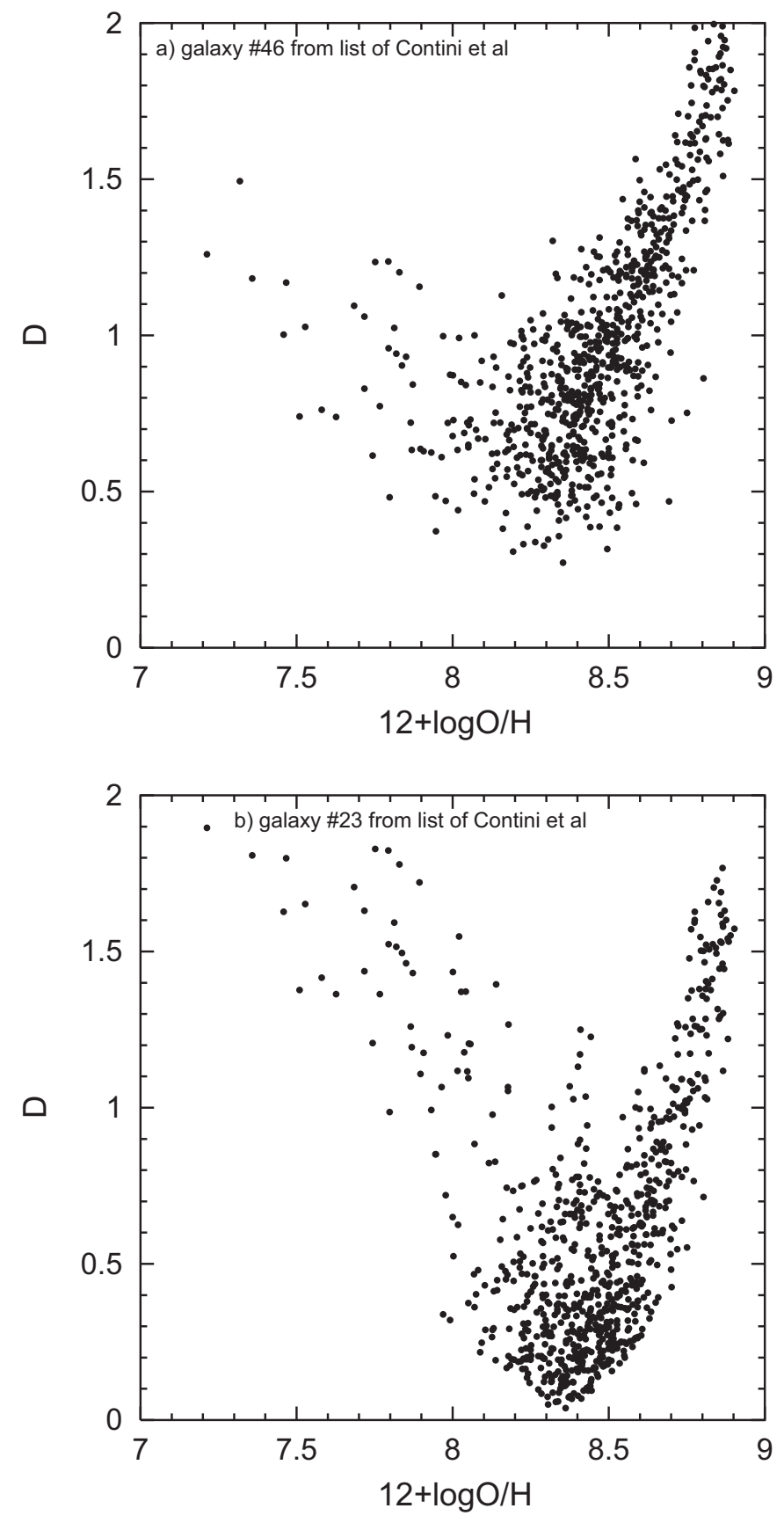

Fig. 11. The difference indexes $D_{j}$ versus the oxygen abundances in individual $\mathrm{H}_{\text {II }}$ regions. Panel a) shows the data for the galaxy \#46 ("peculiar" case) from the list of Contini et al. (2002). For comparison, panel b) shows the data for galaxy \#23 ("normal" case) from the list of Contini et al. (2002).

for $D_{U}$ and $D_{L}$ as a function of $12+\log (\mathrm{O} / \mathrm{H})_{D}$ are quite similar for both samples of galaxies.

Using the conditions listed above, we selected the galaxies from Contini et al. (2002) which are expected to lie on the upper branch of the $\mathrm{O} / \mathrm{H}-R_{23}$ relation. Chemical abundances in these galaxies were determined through the high-metallicity $P$-calibration. The derived abundances are presented in Table 1. The galaxy number according to the list of Contini et al. (2002) is listed in Col. 1. The original oxygen abundance and

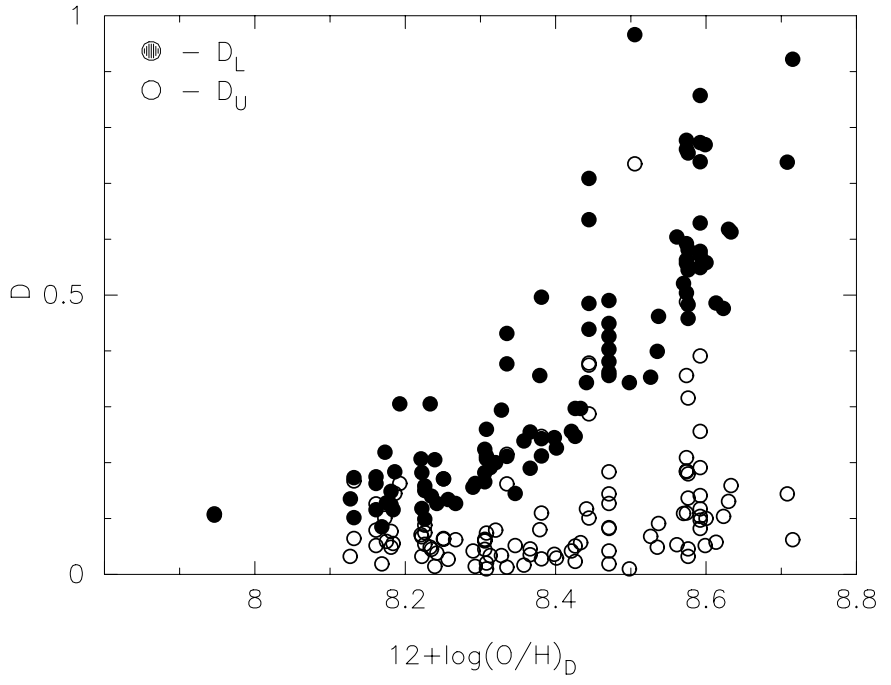

Fig. 12. The difference indexes $D_{U}$ (open circles) and $D_{L}$ (filled circles) as a function of the $12+\log (\mathrm{O} / \mathrm{H})_{D}$ for the sample of nearby galaxies (Jansen et al. 2000).

nitrogen-to-oxygen abundance ratio are given in Cols. 2 and 3 . The oxygen abundance and nitrogen-to-oxygen abundance ratio estimated via the $D$-method are reported in Cols. 4 and 5. The oxygen abundance and nitrogen-to-oxygen abundance ratio determined through the $P$-method for high-metallicity galaxies are listed in Cols. 6 and 7.

It should be stressed that the lower limit of oxygen abundances where the high-metallicity $P$-calibration is suitable for spiral galaxies does not coincide with that for individual $\mathrm{H}_{\text {II }}$ regions. Examination of Figs. 5 and 6 shows that the $P$-calibration for the high-metallicity branch of the $\mathrm{O} / \mathrm{H}-R_{23}$ relation provides realistic global oxygen abundances only for galaxies with a global oxygen abundance higher than $12+\log (\mathrm{O} / \mathrm{H}) \sim 8.3$, while the high-metallicity $P$-calibration provides realistic oxygen abundances in individual $\mathrm{H}$ II regions with $12+\log (\mathrm{O} / \mathrm{H})>8.2$. It is due to the following reason. Spiral galaxies show usually a radial abundance gradient. If a spiral galaxy has a global oxygen abundance in the range $8.2<12+\log (\mathrm{O} / \mathrm{H})<8.3$ then this spiral is populated both by $\mathrm{H}$ II regions with $12+\log (\mathrm{O} / \mathrm{H})>8.2$ and by $\mathrm{H}$ II regions with $12+\log (\mathrm{O} / \mathrm{H})<8.2$. H II regions with $12+\log (\mathrm{O} / \mathrm{H})<8.2$ can make a significant contribution to the global spectrum of such a galaxy. As a result, the high-metallicity $P$-calibration cannot be used for abundance determination in this type of galaxy. Formally, one can say that the boundary between the upper branch and the transition zone in the $\mathrm{O} / \mathrm{H}-R_{23}$ relation for spiral galaxies does not coincide with that for individual $\mathrm{H}$ II regions.

The N/O-O/H diagram for the UV-selected galaxies is presented in Fig. 13. Panel a) shows the original oxygen abundances and nitrogen-to-oxygen abundance ratios from Contini et al. (2002) determined with the calibration of Kobulnicky et al. (1999). The dashed lines outline the area occupied by the individual $\mathrm{H}_{\text {II }}$ regions from our collection (see Fig. 2). The oxygen abundances and nitrogen-to-oxygen abundance ratios estimated in UV-selected galaxies via the $D$-method are presented in panel b). Panel c) shows the oxygen abundances 
Table 1. Oxygen abundances and nitrogen-to-oxygen abundance ratios for the sample of UV-selected galaxies from Contini et al. (2002).

\begin{tabular}{|c|c|c|c|c|c|c|}
\hline $\begin{array}{l}\text { Galaxy } \\
\text { number }\end{array}$ & $\begin{array}{c}12+\log (\mathrm{O} / \mathrm{H}) \\
\text { original } \\
\text { data }\end{array}$ & $\begin{array}{l}\log (\mathrm{N} / \mathrm{O}) \\
\text { original } \\
\text { data }\end{array}$ & $\begin{array}{c}12+\log (\mathrm{O} / \mathrm{H}) \\
D \text {-method }\end{array}$ & $\begin{array}{l}\log (\mathrm{N} / \mathrm{O}) \\
D \text {-method }\end{array}$ & $\begin{array}{c}12+\log (\mathrm{O} / \mathrm{H}) \\
P \text {-method }\end{array}$ & $\begin{array}{l}\log (\mathrm{N} / \mathrm{O}) \\
P \text {-method }\end{array}$ \\
\hline 1 & 8.99 & -1.04 & 8.64 & -0.96 & 8.67 & -1.01 \\
\hline 2 & 8.72 & -1.58 & 8.34 & -1.48 & 8.38 & -1.54 \\
\hline 3 & 8.72 & -1.05 & 8.54 & -0.85 & 8.38 & -0.98 \\
\hline 4 & 8.39 & -0.93 & 7.97 & -0.98 & & \\
\hline 5 & 8.13 & -1.83 & 7.95 & -1.53 & & \\
\hline 6 & 8.66 & -1.13 & 8.39 & -1.00 & 8.25 & -1.04 \\
\hline 7 & 8.20 & -1.57 & 8.17 & -1.52 & & \\
\hline 8 & 8.31 & -1.84 & 7.95 & -1.53 & & \\
\hline 9 & 8.78 & -1.48 & 8.51 & -1.26 & 8.39 & -1.40 \\
\hline 10 & 8.22 & -1.62 & 8.23 & -1.61 & & \\
\hline 11 & 8.02 & -1.29 & 8.48 & -1.39 & 8.47 & -1.40 \\
\hline 12 & 8.38 & -1.60 & 8.24 & -1.43 & & \\
\hline 13 & 8.90 & -0.83 & 8.60 & -0.78 & 8.59 & -0.80 \\
\hline 14 & 8.45 & -1.18 & 8.13 & -1.07 & & \\
\hline 15 & 8.69 & -1.23 & 8.51 & -1.24 & 8.47 & -1.28 \\
\hline 16 & 8.80 & -1.08 & 8.51 & -1.10 & 8.50 & -1.06 \\
\hline 17 & 8.35 & -1.74 & 8.16 & -1.65 & & \\
\hline 18 & 8.05 & -1.73 & 8.27 & -1.62 & & \\
\hline 19 & 8.63 & -1.22 & 8.33 & -1.15 & 8.31 & -1.20 \\
\hline 20 & 8.66 & -1.12 & 8.35 & -1.09 & 8.34 & -1.10 \\
\hline 21 & 8.49 & -1.26 & 8.17 & -1.26 & & \\
\hline 22 & 8.72 & -1.20 & 8.33 & -1.08 & 8.35 & -1.14 \\
\hline 23 & 8.65 & -1.15 & 8.36 & -1.13 & 8.36 & -1.14 \\
\hline 24 & 8.50 & -0.91 & 8.16 & -0.93 & & \\
\hline 25 & 8.45 & -1.01 & 8.29 & -1.09 & & \\
\hline 26 & 8.36 & -1.51 & 8.07 & -1.50 & & \\
\hline 27 & 8.62 & -1.05 & 8.34 & -1.01 & 8.34 & -1.04 \\
\hline 28 & 8.42 & -1.25 & 8.20 & -1.12 & & \\
\hline 29 & 8.73 & -0.86 & 8.40 & -0.90 & 8.41 & -0.84 \\
\hline 30 & 7.86 & -1.78 & 7.95 & -1.53 & & \\
\hline 31 & 8.08 & -1.79 & 8.27 & -1.62 & & \\
\hline 32 & 8.06 & -1.71 & 8.27 & -1.62 & & \\
\hline 33 & 8.36 & -1.44 & 8.05 & -1.33 & & \\
\hline 34 & 8.36 & -1.52 & 8.07 & -1.50 & & \\
\hline 35 & 8.35 & -1.67 & 8.02 & -1.63 & & \\
\hline 36 & 8.84 & -1.25 & 8.56 & -1.07 & 8.45 & -1.18 \\
\hline 37 & 8.50 & -1.20 & 8.13 & -1.07 & & \\
\hline 38 & 8.63 & -1.32 & 8.24 & -1.25 & & \\
\hline 39 & 8.36 & -1.54 & 8.10 & -1.50 & & \\
\hline 40 & 8.61 & -1.35 & 8.23 & -1.28 & & \\
\hline 41 & 8.37 & -1.53 & 8.10 & -1.50 & & \\
\hline 42 & 8.77 & -1.19 & 8.51 & -1.15 & 8.51 & -1.21 \\
\hline 43 & 8.35 & -1.65 & 8.02 & -1.63 & & \\
\hline 44 & 8.15 & -1.74 & 7.95 & -1.53 & & \\
\hline 45 & 8.38 & -1.47 & 8.11 & -1.35 & & \\
\hline 46 & 7.99 & -1.82 & 8.35 & -1.63 & 8.41 & -1.86 \\
\hline 47 & 8.46 & -1.19 & 8.39 & -1.25 & 8.36 & -1.30 \\
\hline 48 & 8.50 & -1.22 & 8.24 & -1.20 & & \\
\hline 49 & 8.38 & -1.48 & 8.11 & -1.35 & & \\
\hline 50 & 8.78 & -1.10 & 8.44 & -1.00 & 8.40 & -1.03 \\
\hline 51 & 8.72 & -1.16 & 8.33 & -1.08 & 8.38 & -1.12 \\
\hline 52 & 7.86 & -1.49 & 8.53 & -1.46 & 8.51 & -1.55 \\
\hline 53 & 7.78 & -1.34 & 8.53 & -1.46 & 8.61 & -1.45 \\
\hline 54 & 8.68 & -1.13 & 8.39 & -1.00 & 8.28 & -1.05 \\
\hline 55 & 8.38 & -1.54 & 8.24 & -1.43 & & \\
\hline 56 & 8.73 & -1.29 & 8.38 & -1.23 & 8.42 & -1.27 \\
\hline 57 & 8.67 & -1.23 & 8.39 & -1.00 & 8.25 & -1.14 \\
\hline 58 & 8.90 & -1.29 & 8.56 & -1.07 & 8.53 & -1.23 \\
\hline 59 & 8.85 & -1.11 & 8.56 & -1.07 & 8.48 & -1.06 \\
\hline 60 & 8.05 & -1.53 & 8.34 & -1.52 & 8.34 & -1.54 \\
\hline 61 & 8.03 & -1.75 & 7.95 & -1.53 & & \\
\hline 62 & 8.94 & -1.17 & 8.63 & -1.10 & 8.65 & -1.16 \\
\hline 63 & 8.72 & -1.19 & 8.39 & -1.00 & 8.33 & -1.12 \\
\hline 64 & 8.82 & -1.36 & 8.51 & -1.26 & 8.47 & -1.33 \\
\hline 65 & 8.73 & -1.40 & 8.34 & -1.48 & 8.41 & -1.38 \\
\hline 66 & 8.36 & -1.50 & 8.05 & -1.33 & & \\
\hline 67 & 8.91 & -1.08 & 8.63 & -1.10 & 8.60 & -1.06 \\
\hline 68 & 8.81 & -1.28 & 8.51 & -1.26 & 8.43 & -1.22 \\
\hline
\end{tabular}



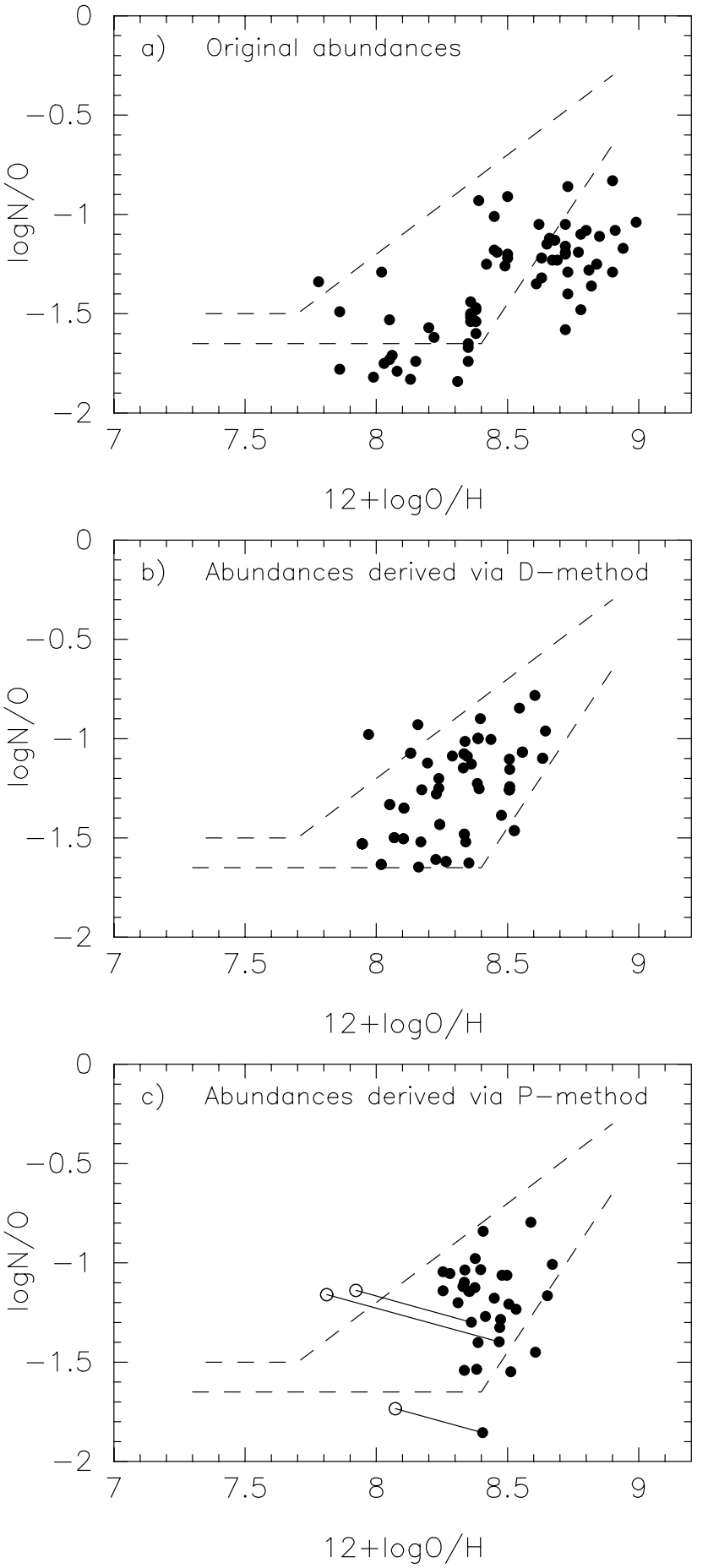

Fig. 13. The N/O vs. O/H diagram for the sample of UV-selected galaxies from Contini et al. (2002). The dashed lines outline the area occupied by individual $\mathrm{H}_{\text {II }}$ regions from our collection (see Fig. 2). a) Filled circles are original abundances from Contini et al. (2002) determined via the calibration of Kobulnicky et al. (1999). b) Filled circles are abundances estimated using the $D$-method (see text). c) Filled circles are abundances in galaxies with $12+\log (\mathrm{O} / \mathrm{H})_{D}>8.3$ determined via the high-metallicity $P$-calibration. The open circles are abundances determined through the low-metallicity $P$-calibration in three galaxies with unreliable classification. The abundances derived via the high- and low-metallicity $P$-calibrations for the same galaxy are connected with a solid line.

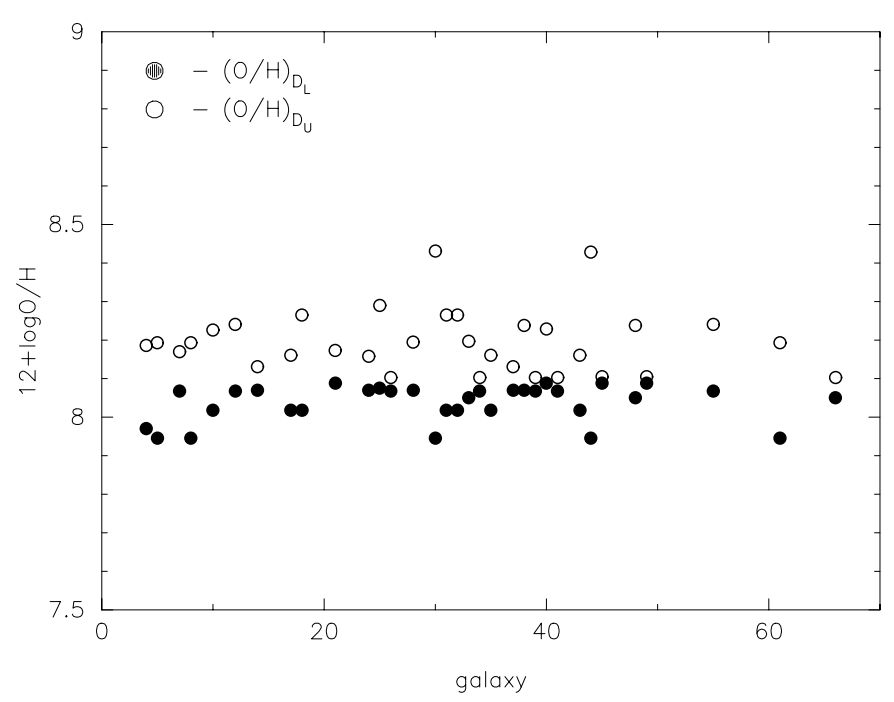

Fig. 14. The oxygen abundances in galaxies with $12+\log (\mathrm{O} / \mathrm{H})_{D}<$ 8.3. The open circles are the oxygen abundances derived through the $D$-method with the $D_{U}$ values, i.e. under the assumption that the galaxy has an oxygen abundance higher than $12+\log (\mathrm{O} / \mathrm{H})=8.1$. The value of $D_{\min }=D_{U}$ is determined by comparing the spectra of galaxies with the spectra of $\mathrm{H}_{\text {II }}$ regions with $12+\log (\mathrm{O} / \mathrm{H})>8.1$. The filled circles are the oxygen abundances derived through the $D$ method with the $D_{L}$ value, i.e. under the assumption that the galaxy has an oxygen abundance lower than $12+\log (\mathrm{O} / \mathrm{H})=8.1$ and the value of $D_{\min }=D_{L}$ is determined by comparing the spectra of galaxies with the spectra of $\mathrm{H}_{\text {II }}$ regions with $12+\log (\mathrm{O} / \mathrm{H})<8.1$.

and nitrogen-to-oxygen abundance ratios determined through the $P$-method for high-metallicity galaxies selected according to the criteria listed above. The comparison of panel a) and panel c) of Fig. 13 shows that the N/O-O/H diagram for highmetallicity $(12+\log (\mathrm{O} / \mathrm{H})>8.3) \mathrm{UV}$-selected galaxies based on the abundances determined through the $P$-method differs significantly from that based on the original abundances derived by Contini et al. (2002). The UV-selected galaxies with abundances determined using the $P$-method occupy the same area as the individual $\mathrm{H}$ II regions in normal spiral galaxies. The shift of original positions of UV-selected galaxies could thus be due to calibration problems for abundance determination.

How many high-metallicity $(12+\log (\mathrm{O} / \mathrm{H})>8.3)$ galaxies are lost using the selection criteria listed above? Indeed, the typical value of $D_{U}$ for low-metallicity galaxies is close to that for high-metallicity galaxies (see Fig. 10). Then, one can expect that the high-metallicity galaxies can exist among the galaxies classified as low-metallicity ones, with $12+$ $\log (\mathrm{O} / \mathrm{H})<8.3$. Figure 14 shows the two values of oxygen abundance in galaxies classified as low-metallicity. The open circles correspond to the oxygen abundances derived through the $D$-method with the $D_{U}$ value, i.e. under the assumption that the galaxy has an oxygen abundance higher than $12+\log (\mathrm{O} / \mathrm{H})=8.1$ and the value of $D_{\min }=D_{U}$ is determined by comparing the spectra of galaxies with the spectra of $\mathrm{H}_{\text {II }}$ regions with $12+\log (\mathrm{O} / \mathrm{H})>8.1$. The filled circles correspond to the oxygen abundances derived through the $D$-method with the $D_{L}$ value, i.e. under the assumption that the galaxy has an oxygen abundance lower than $12+\log (\mathrm{O} / \mathrm{H})=8.1$ 
and the value of $D_{\min }=D_{L}$ is determined by comparing the spectra of galaxies with the spectra of $\mathrm{H}_{\text {II }}$ regions with $12+\log (\mathrm{O} / \mathrm{H})<8.1$. Examination of Fig. 14 shows that both oxygen abundances $12+\log (\mathrm{O} / \mathrm{H})_{L}$ and oxygen abundances $12+\log (\mathrm{O} / \mathrm{H})_{U}$ in galaxies classified as low-metallicity ones are below $12+\log (\mathrm{O} / \mathrm{H})=8.3$, with two exceptions. The classification of only two galaxies, \#30 and \#44, may be wrong. The bulk of galaxies classified as low-metallicity ones appear to belong to the transition zone of the $\mathrm{O} / \mathrm{H}-R_{23}$ relation, or some of them lie on the lower branch of this relation. Thus, the selection criterion used in this study seems to be reliable.

The typical difference between oxygen abundance determined in high-metallicity galaxies via the $P$-method and that estimated via the $D$-method is small, i.e. $<0.1$ dex (see Table 1 ). Since the values of $D_{\min }$ for low-metallicity galaxies are similar to those for high-metallicity galaxies (see Fig. 10), one can hope that the $D$-method also provides realistic estimations of the oxygen abundance in low-metallicity galaxies which belong to the transition zone or lie on the lower branch of the $\mathrm{O} / \mathrm{H}-R_{23}$ relation. It should be noted that the oxygen abundance in our collection of individual $\mathrm{H}_{\text {II }}$ regions with $12+$ $\log (\mathrm{O} / \mathrm{H})<8.2$ was determined with the $T_{\mathrm{e}}$-method. Panel b) of Fig. 13 shows that the low-metallicity UV-selected galaxies fill more or less uniformily the area outlined by the individual $\mathrm{H}$ II regions in the $\mathrm{N} / \mathrm{O}-\mathrm{O} / \mathrm{H}$ diagram, i.e. the low-metallicity UV-selected galaxies do not show any shift relative to the positions of individual $\mathrm{H}_{\text {II }}$ regions in local spiral galaxies.

The fact that the typical difference between $\mathrm{O} / \mathrm{H}$ determined with the $P$-method and that estimated via the $D$-method is small (see Table 1) shows that our H II region spectral database is large enough so that any spectrum of UV-selected galaxies considered in this study can be represented by one of these $\mathrm{H}$ II region spectra.

\section{The luminosity-metallicity relation}

The luminosity-metallicity relation for UV-selected local and intermediate-redshift $(0<z \leq 0.4)$ galaxies of Contini et al. (2002) is presented in Fig. 15. The upper panel a) shows the original data. The luminosity-metallicity relation with oxygen abundances re-determined via the $D$-method is shown in the lower panel. The global optical spectra for 14 starforming emission-line galaxies at intermediate redshifts $(0.1<$ $z<0.5)$ have been published by Kobulnicky \& Zaritsky (1999). The oxygen abundances in five galaxies with mea-

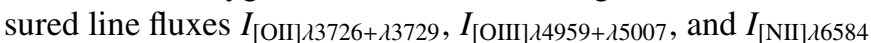
(L2-408115, L2-410083, SA 68-206134, SDG 223, and SA 68-207213) from the sample of Kobulnicky \& Zaritsky (1999) have been estimated here via the $D$-method. The position of these galaxies in the luminosity-metallicity diagram is also shown (filled squares) in the lower panel of Fig. 15. To compare the luminosity-metallicity relation for the samples of galaxies from Kobulnicky \& Zaritsky (1999) and from Contini et al. (2002), the absolute magnitudes of galaxies from Kobulnicky \& Zaritsky (1999) have been decreased by $-1.5 \mathrm{mag}$ since they used the value of $H_{0}=$ $50 \mathrm{~km} \mathrm{~s}^{-1} \mathrm{Mpc}^{-1}$ while the absolute magnitude for galaxies of Contini et al. were estimated with $H_{0}=100 \mathrm{~km} \mathrm{~s}^{-1} \mathrm{Mpc}^{-1}$. The
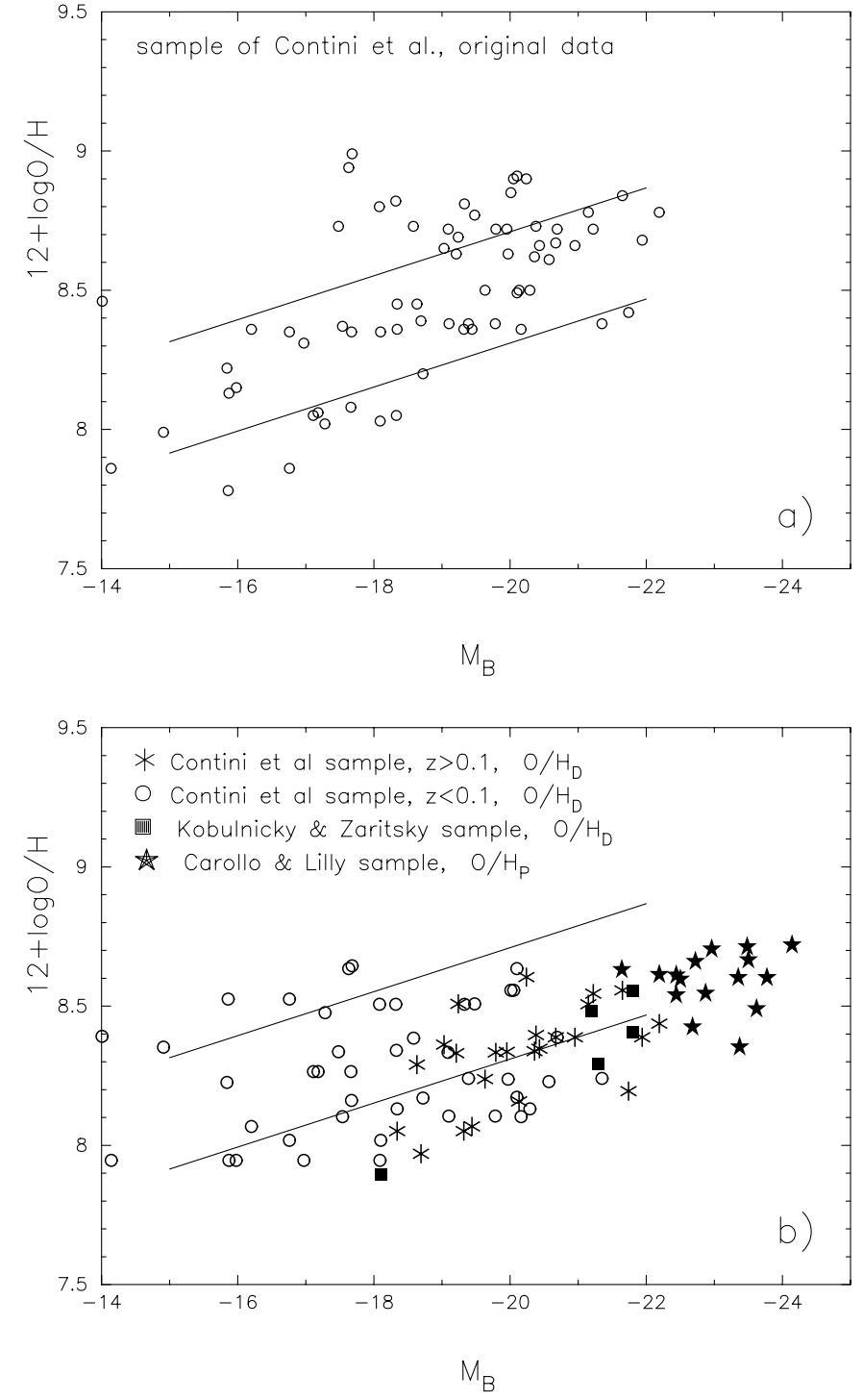

Fig. 15. Luminosity-metallicity relation. a) Open circles are the original data for the sample of UV-selected local and intermediate-redshift galaxies from Contini et al. (2002). The solid lines correspond to the "luminosity-metallicity band" occupied by well-studied local spiral galaxies (Paper I). b) The luminosity-metallicity relation for the the Contini et al.'s sample of UV-selected local $(z<0.1$; open circles) and intermediate-redshift $(z>0.1$; asterisks) galaxies with oxygen abundances re-determined via the $D$-method. The filled squares are the intermediate-redshift $(0.1<z<0.5)$ galaxies from the sample of Kobulnicky \& Zaritsky (1999) with oxygen abundances rederived via the $D$-method and absolute blue magnitudes scaled to the value of $H_{0}=100 \mathrm{~km} \mathrm{~s}^{-1} \mathrm{Mpc}^{-1}$. The stars are intermediate-redshift $(0.5<z<1.0)$ galaxies from the sample of Carollo \& Lilly (2001) with oxygen abundances derived via the $P$-method and absolute blue magnitudes scaled to the value of $H_{0}=100 \mathrm{~km} \mathrm{~s}^{-1} \mathrm{Mpc}^{-1}$.

solid lines in Fig. 15 outline the "luminosity-metallicity band" occupied by well-studied local spiral galaxies (Paper I).

Figure $15 \mathrm{~b}$ shows that the UV-selected intermediateredshift sample $(z>0.1)$, as well as the sample of intermediate-redshift galaxies from Kobulnicky \& Zaritsky (1999), have, on average, lower oxygen abundances compared to the local galaxies of the same luminosity or/and that the 
intermediate-redshift galaxies are slightly more luminous than local galaxies of the same metallicity. This does not agree with the conclusions of Carollo \& Lilly (2001). They measured the emission-line flux ratios in global spectra of intermediateredshift $(0.5<z<1.0)$ galaxies. Based on the similarity between the positions of these galaxies and that of local field galaxies from the sample of Jansen et al. (2000) in the $R_{23}-[\mathrm{OIII}] /[\mathrm{OII}]$ and the $R_{23}-M_{B}$ diagrams, they concluded that the metallicities of the intermediate-redshift galaxies appear to be remarkably similar to those of local field galaxies, and there appears to have been little change in the relationship between metallicity and luminosity from $z \sim 1$ to today. It should be noted that the absolute blue magnitudes of galaxies in these samples are estimated with different values of $H_{0}$; with $H_{0}=50 \mathrm{~km} \mathrm{~s}^{-1} \mathrm{Mpc}^{-1}$ in the sample of Carollo \& Lilly (2001) and with $H_{0}=100 \mathrm{~km} \mathrm{~s}^{-1} \mathrm{Mpc}^{-1}$ in the sample of Jansen et al. (2000). Then the direct comparison of the $R_{23}-M_{B}$ diagram for these samples of galaxies is not justified. The oxygen abundances in 13 galaxies (two AGNs were excluded from our consideration) from the sample of Carollo \& Lilly (2001) with measured line fluxes as well as in 3 galaxies with estimated upper limits of [OIII] $\lambda \lambda 4959,5007$ line fluxes were re-determined using the $P$-method ${ }^{2}$. The absolute magnitudes of galaxies from Carollo \& Lilly (2001) have been decreased by $-1.5 \mathrm{mag}$ since they used the value of $H_{0}=50 \mathrm{~km} \mathrm{~s}^{-1} \mathrm{Mpc}^{-1}$ while the absolute magnitude for galaxies of Contini et al. have been estimated with $H_{0}=100 \mathrm{~km} \mathrm{~s}^{-1} \mathrm{Mpc}^{-1}$. The $\mathrm{O} / \mathrm{H}-M_{B}$ diagram for the sample of Carollo \& Lilly (2001) is presented in lower panel of Fig. 15 by stars. It is clear that the intermediate-redshift galaxies from Carollo \& Lilly fall in the $\mathrm{O} / \mathrm{H}$ versus $M_{B}$ plane defined by other intermediate-redshift galaxies and show a shift relative to the local field galaxy sample.

It should be noted however that the luminosity of intermediate-redshift galaxies has been estimated using the galaxy redshifts. As a consequence, the luminosity of intermediate- $z$ galaxies depends on the adopted value of the Hubble constant $H_{0}$, while accurate distance determinations with high-precision methods (the Cepheid period-luminosity relation, the peak brightness of type Ia supernovae, the expanding photospheres of type II supernovae and others) are available for the majority of local spiral galaxies (see Paper I). The absolute blue luminosity of local galaxies does not depend on the value of the Hubble constant $H_{0}$. Could the shift in the positions of the intermediate-redshift galaxies in the luminositymetallicity diagram relative to the positions of the local spiral galaxies be due to the choice (somewhat arbitrary) of the Hubble constant value?

Jansen et al. (2000) published global spectra for a representative sample of nearby galaxies. The oxygen abundances in nearby galaxies with measured global line

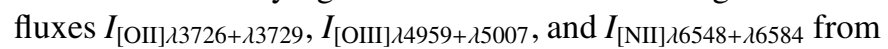
the sample of Jansen et al. (2000) were estimated via the $D$-method. The luminosity-metallicity diagram for the sample

\footnotetext{
${ }^{2}$ The $D$-method cannot be used for the abundance determination in Carollo \& Lilly's galaxies since the [NII] 26584 line measurements are not available.
}

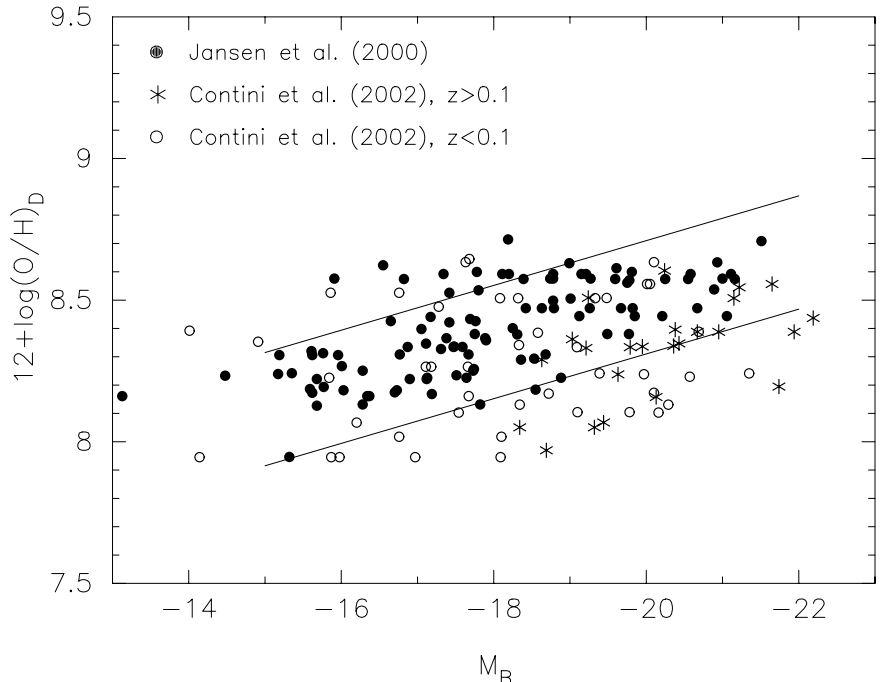

Fig. 16. Luminosity-metallicity relations for the sample of nearby galaxies (filled circles) from Jansen et al. (2000) and for the sample of UV-selected local ( $z<0.1$; open circles) and intermediate-redshift $(z>0.1$; asterisks) galaxies from Contini et al. (2002). The solid lines show the "luminosity-metallicity band" obtained in Paper I for wellstudied local spirals.

of nearby galaxies from Jansen et al. (2000) is shown in Fig. 16. Examination of this figure shows that the local galaxies from the sample of Jansen et al. (2000) occupy the same band in the luminosity-metallicity diagram as local galaxies from the sample of Paper I. The absolute blue magnitudes for these galaxies have been calculated by Jansen et al. from the total apparent $B$-band magnitude and the galaxy redshifts, assuming a simple Hubble flow and $H_{0}=100 \mathrm{~km} \mathrm{~s}^{-1} \mathrm{Mpc}^{-1}$. Therefore the luminosity-metallicity relation for the sample of Jansen et al. (2000) can be directly compared to the luminosity-metallicity relation for the sample of Contini et al. (2002).

In Fig. 16, we compare the luminosity-metallicity relations for the sample of Contini et al. (2002) and Jansen et al. (2000). It shows that a significant fraction of UV-selected galaxies are below the luminosity-metallicity defined by normal nearby galaxies. Thus, the shift in the position of the intermediateredshift galaxies in the luminosity-metallicity diagram relative to the position of the nearby galaxies seems to be real and does not depend on the choice of the Hubble constant value.

It thus appears that the intermediate-redshift galaxies systematically deviate from the metallicity-luminosity trend of local galaxies. One interpretation could be that the intermediate-redshift galaxies are slightly less advanced in their chemical evolution and, as a consequence, are more metal-deficient than nearby galaxies. Another interpretation could be that intermediate- $z$ galaxies are observed at a special stage in their evolution; they have just undergone a powerful starburst which temporary lowered their mass-to-light ratio, resulting in a temporary increase of their blue luminosity.

If intermediate-redshift galaxies are slightly less advanced in their evolution, one can expect that the deviation from the local $L-Z$ relation correlates with redshift. The deviation of oxygen abundance from the local metallicity-luminosity relation was estimated in the following way. Taking into account $i$ ) that the $H_{0}$-dependent luminosity-metallicity relation 


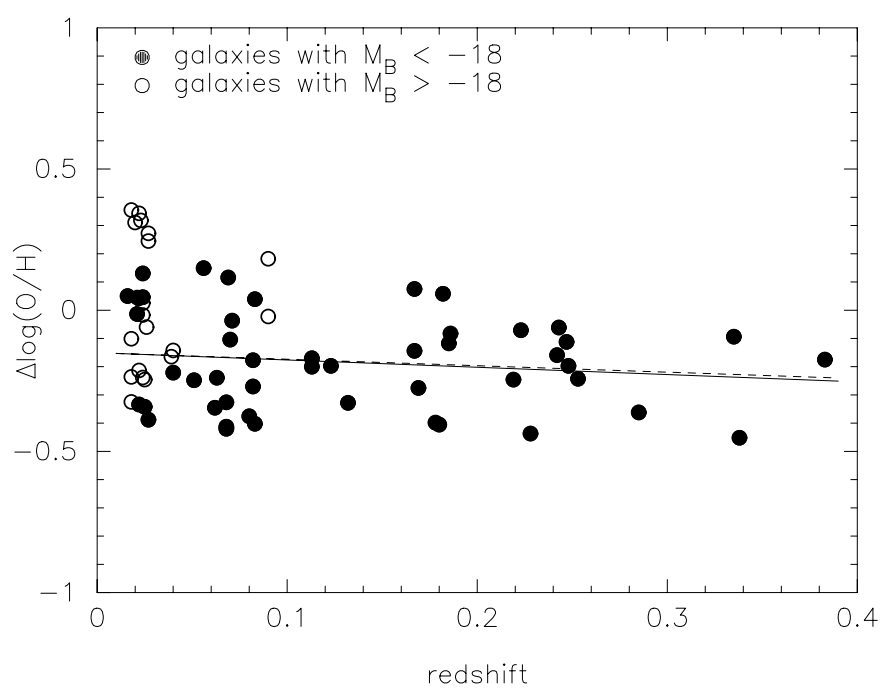

Fig. 17. The deviation of the oxygen abundance from the general luminosity-metallicity trend (obtained in Paper I) as a function of redshift for the sample of UV-selected galaxies from Contini et al. (2002). The filled circles are galaxies with $M_{B}<-18$, the open circles are galaxies with $M_{B}>-18$. The solid line corresponds to the best fit to the data for galaxies with $M_{B}<-18$, the dashed line corresponds to the best fit to galaxies with redshift $z>0.1$.

for local galaxies (Jansen et al. 2000) is in good agreement with the $H_{0}$-independent $L-Z$ relation for the well-studied local galaxies of Paper I (see Fig. 16), and ii) that absolute blue magnitudes for UV-selected and nearby galaxies have been calculated with the same value of $H_{0}$, the metallicity-luminosity relationship from Paper I,

$12+\log (\mathrm{O} / \mathrm{H})=6.93-0.079 M_{B}$,

can be used to estimate the deviation of oxygen abundance from the metallicity-luminosity trend. Then, the value of the oxygen abundance deviation is $\Delta \log (\mathrm{O} / \mathrm{H})=(12+$ $\left.\log (\mathrm{O} / \mathrm{H})_{D}\right)-\left(12+\log (\mathrm{O} / \mathrm{H})^{*}\right)$, where $12+\log (\mathrm{O} / \mathrm{H})^{*}$ is estimated from the absolute blue magnitude using Eq. (6). The deviation of the oxygen abundance from the general luminositymetallicity trend as a function of redshift for the sample of UV-selected galaxies from Contini et al. (2002) is presented in Fig. 17. The filled circles are galaxies with $M_{B}<-18$, the open circles are galaxies with $M_{B}>-18$. Examination of Fig. 17 shows that the dispersion of points increases with decreasing redshift and reaches a maximum value near $z=0$. This could be due to the fact that the distance and consequently the absolute blue magnitude for galaxies calculated from the galaxy redshifts are less accurate at low redshifts since peculiar motions can make a significant contribution to the observed values of $z$. On the other hand, close examination of Fig. 17 shows that the low-luminosity galaxies, with $M_{B}>-18$, have a larger dispersion than luminous ones. It can indicate that the emission-line intensity measurements are more accurate in luminous than in low-luminosity galaxies.

The solid line in Fig. 17 corresponds to the best fit to the data for galaxies with $M_{B}<-18$, the dashed line corresponds to the best fit to galaxies with redshift $z>0.1$. Both relations show that the position of local $(z \sim 0) \mathrm{UV}$-selected galaxies are shifted from the luminosity-metallicity trend of normal galaxies. It suggests that a temporary increase of their blue luminosity could contribute significantly to the shift of UV-selected galaxies in the luminosity-metallicity diagram.

Figure 17 shows that there is a marginal correlation (correlation coefficient $r<0.1$ ) between the deviation of oxygen abundance in a galaxy from the metallicity-luminosity trend and galaxy redshift. If one tries to coincide the positions of UV-selected galaxies at $z=0$ with the position of normal galaxies by shifting the UV-selected galaxies by $\sim 0.15$ dex in the vertical direction, one can find deviations of oxygen abundance of intermediate-redshift $(z \sim 0.4)$ galaxies as large as $\sim 0.1$ dex (see Fig. 17) if the correlation is real. This could suggest that intermediate-redshift galaxies are slightly less advanced in their evolution and, as a consequence, are more metal-deficient (by $\sim 0.1 \mathrm{dex}$ ) than local galaxies. However, since the dispersion of points in Fig. 17 is large (the mean value of deviation $\Delta \log (\mathrm{O} / \mathrm{H}) \sim 0.15 \mathrm{dex})$ and the correlation is very weak, no firm conclusion can be drawn from this study on the evolution with redshift of the oxygen abundance of galaxies.

\section{Conclusions}

The validity of oxygen and nitrogen abundances derived via the $P$-method from the global spectra of galaxies has been investigated using a collection of published spectra of individual $\mathrm{H}_{\text {II }}$ regions in irregular and spiral galaxies. It has been shown that the oxygen abundance derived from the global emissionline spectrum of high-metallicity galaxies using the $P$-method agrees very well with the oxygen abundance at galactocentric distance ( $\left.r=0.4 R_{25}\right)$, traced by individual $\mathrm{H}$ II regions, if most of $\mathrm{H}$ II regions belong to the upper branch of the $\mathrm{O} / \mathrm{H}-R_{23}$ relation. The oxygen abundances in low-metallicity galaxies derived from the global emission-line spectra via the $P$-method are slightly underestimated, by $\Delta(\mathrm{O} / \mathrm{H}) \leq 0.1 \mathrm{dex}$. We thus confirm the conclusion of Kobulnicky et al. (1999) that global emission-line spectra can reliably indicate the chemical properties of galaxies.

It has been shown that the comparison of the global spectrum of a galaxy with a collection of spectra of individual $\mathrm{H}$ II regions can be used to distinguish high- versus lowmetallicity objects, i.e. to establish whether the galaxy belongs to the upper or lower branch of the $\mathrm{O} / \mathrm{H}-R_{23}$ relation. This method (called the $D$-method) can further be used for the determination of accurate abundances in a galaxy.

The oxygen and nitrogen abundances in a sample of UV-selected local and intermediate-redshift galaxies from Contini et al. (2002) have been determined using both the $D$ method and the $P$-method. It has been found that the UVselected galaxies fill more or less uniformly the area outlined in the $\mathrm{N} / \mathrm{O}-\mathrm{O} / \mathrm{H}$ diagram by individual $\mathrm{H}$ II regions of wellstudied local galaxies. The UV-selected galaxies do not show any significant shift in the $\mathrm{N} / \mathrm{O}-\mathrm{O} / \mathrm{H}$ diagram relative to the individual $\mathrm{H}$ II regions in "normal" local galaxies.

Finaly, it has been found that the intermediate-redshift galaxies seem to systematically deviate from the metallicityluminosity trend of local galaxies. One explanation could be that intermediate-redshift galaxies are slightly less advanced 
in their evolution and, as a consequence, are slightly more metal-deficient than local galaxies of the same luminosity. As an alternative, intermediate-redshift galaxies may have just undergone a powerful starburst which temporary increases their blue luminosity. Given the limited size of the present samples of intermediate-redshift galaxies, no firm conclusion can be drawn from this study on the evolution with redshift of the oxygen abundance of galaxies. No doubt, on-going deep spectroscopic surveys, such as VVDS or DEEP, will shed new light on this important issue.

Acknowledgements. We are grateful to the anonymous referee for making constructive suggestions to improve the paper. L.S.P thanks the Laboratoire d'Astrophysique de l'Observatoire Midi-Pyrénées (UMR 5572) for their hospitality during his visit where this work was done. This study was supported (LSP) by the Laboratoire d'Astrophysique de l'Observatoire Midi-Pyrénées (UMR 5572) within the framework of "postes rouges" and by the Ukrainian Fund of Fundamental Investigation, grant No. 02.07/00132. J.V.M. was supported by project AYA2001-3939-03-C01 of the spanish Programa Nacional de Astronomia y Astrofisica of the MCyT.

\section{References}

Carollo, C. M., \& Lilly, S. J. 2001, ApJ, 548, L153

Contini, T., Treyer, M. A., Sullivan, M., \& Ellis, R. S. 2002, MNRAS, 330,75

Dopita, M. A., \& Evans, I. N. 1986, ApJ, 307, 431

Edmunds, M. G., \& Pagel, B. E. J. 1984, MNRAS, 211, 507

Garnett, D. R. 1992, AJ, 103, 1330

Garnett, D. R. 2002, ApJ, 581, 1019

Garnett, D. R., \& Shields, G. A. 1987, ApJ, 317, 82
Jansen, R. A., Fabricant, D., Franx, M., \& Caldwell, N. 2000, ApJS, 126,331

Kennicutt, R. C. 1992, ApJ, 388, 310

Kobulnicky, H. A., Kennicutt, R. C., \& Pizagno, J. L. 1999, ApJ, 514, 544

Kobulnicky, H. A., \& Zaritsky, D. 1999, ApJ, 511, 118

Lamareille, F., Mouhcine, M., Contini, T., Lewis, I., \& Maddox, S. 2004, MNRAS, 350, 396

McCall, M. L., Rybski, P. M., \& Shields, G. A. 1985, ApJS, 57, 1

McGaugh, S. S. 1991, ApJ, 380, 140

Melbourne, J., \& Salzer, J. J. 2002, AJ, 123, 2302

Melbourne, J., Phillips, A., Salzer, J. J., Gronwall, C., \& Sarajedini, V. L. 2004, AJ, 127, 686

Mouhcine, M., \& Contini, T. 2002, A\&A, 389, 106

Pagel, B. E. J., Edmunds, M. G., Blackwell, D. E., Chun, M. S., \& Smith, G. 1979, MNRAS, 189, 95

Pilyugin, L. S. 2000, A\&A, 362, 325

Pilyugin, L. S. 2001a, A\&A, 369, 594

Pilyugin, L. S. 2001b, A\&A, 373, 56

Pilyugin, L. S. 2001c, A\&A, 374, 412

Pilyugin, L. S. 2003, A\&A, 399, 1003

Pilyugin, L. S., Thuan, T. X., \& Vílchez, J. M. 2003, A\&A, 397, 487

Pilyugin, L. S., Vílchez, J. M., \& Contini, T. 2004, A\&A, submitted (Paper I)

Rozas, M., Knapen, J. H., \& Beckman, J. E. 1996, A\&A, 312, 275

Rozas, M., Zurita, A., Heller, C. H., \& Beckman, J. E. 1999, A\&AS, 135,145

Tremonti, C. A., Heckman, T. N., Kauffmann, G., et al. 2003, IAU Symp., 217, in press

Vila-Costas, M. B., \& Edmunds, M. G. 1992, MNRAS, 259, 121

Wegner, G., Salzer, J. J., Jangren, A., Gronwall, C., \& Melbourne, J. 2003, AJ, 125, 2373

Zaritsky, D., Kennicutt, R. C., \& Huchra, J. P. 1994, ApJ, 420, 87 